\title{
Four-dimensional Electron Microscopy: Ultrafast Imaging, Diffraction and Spectroscopy in Materials Science and Biology
}

\author{
G. M. Vanacore ${ }^{\ddagger}$, A. W. P. Fitzpatrick ${ }^{\dagger}$, and A. H. Zewail ${ }^{*}$ \\ Physical Biology Center for Ultrafast Science and Technology, Arthur Amos Noyes \\ Laboratory of Chemical Physics, California Institute of Technology, Pasadena, California \\ 91125, United States.
}

\begin{abstract}
Understanding the ultrafast evolution of atomic and electronic rearrangements under nonequilibrium conditions in organic, inorganic and biological materials plays a fundamental role in deciphering the mechanism governing chemical and biological functions. With direct visualization, the technological development of future innovative devices on the nanoscale becomes feasible.

Although an enormous effort has been devoted to the comprehension and improvement of these materials and devices, the capability of investigating their dynamic behavior is hindered by the difficulty of simultaneously studying their evolution in space and time at the appropriate scales. The traditional characterization techniques and the steady-state theoretical models

\footnotetext{
${ }^{\ddagger}$ Current address: Institute of Condensed Matter Physics, École Polytechnique Fédérale de Lausanne, CH-1015 Lausanne, Switzerland.

${ }^{\dagger}$ Current address: Department of Chemistry, University of Cambridge, Cambridge CB2 1EW, United Kingdom.

${ }^{*}$ Corresponding author. E-mail address: zewail@caltech.edu
}

March 9, 2016

(C) 2016. This manuscript version is made available under the Elsevier user license http://www.elsevier.com/open-access/userlicense/1.0/ 
are both not adequate for describing their nonequilibrium behavior. Instead, a novel approach for visualization of matter with high temporal and spatial resolutions, together with energy and momentum selection, is indispensable to fully exploit their potential.

Four-dimensional electron microscopy (4D-EM) has been developed with the capability of performing time-resolved imaging, diffraction and electronspectroscopy. The acquired and sufficient spatial, temporal, energy and momentum resolution, provide the real-time access to the dynamic behavior of surfaces, interfaces and nanosystems.

In this review, after outlining the 4D-EM approach, which enables subnanometer spatial resolution and temporal speed of 10 orders of magnitude faster than previously possible, we address several recent applications in materials science and biology, highlighting for each case the challenges that had to be overcome, the main scientific contributions, and future trends in the field. The highly inter- and multi-disciplinary approach presented here will pave the way for an unprecedented insight into the nonequilibrium phenomena of advanced materials, and should play a decisive role in the rational design and engineering of future applications.

Keywords: electron microscopy, electron diffraction, electron energy loss spectroscopy, ultrafast phenomena, phonons, plasmonics, PINEM, nanofriction, phase transitions, carrier transport, amyloid, DNA nanostructures, cryo-electron microscopy, materials science, biology 


\section{Table of Contents}

1 Introduction $\quad 5$

2 Basic concepts $\quad 8$

2.1 Ultrafast diffraction . . . . . . . . . . . . . . . . . 8

2.2 Ultrafast microscopy of single particles . . . . . . . . . . . . . 9

2.3 Ultrafast electron-spectroscopy . . . . . . . . . . . . . . . . 10

2.4 Scanning ultrafast microscopy . . . . . . . . . . . . . . . 11

3 Applications: Materials Science 12

3.1 Nanoscale acoustic phonon dynamics . . . . . . . . . . . 13

3.2 Nanoplasmonics and Nanophotonics . . . . . . . . . . . . 17

3.3 Nanofriction . . . . . . . . . . . . . . . . 21

3.4 Phase transitions . . . . . . . . . . . . . . . . 23

3.5 Carrier transport ................. . 27

3.6 Valence and core-level excitation . . . . . . . . . . . . . . 29

4 Applications: Biology 31

4.1 PINEM imaging of biological specimens . . . . . . . . . . . . . 32

4.24 D cryo-electron microscopy . . . . . . . . . . . . . . 34

4.3 Biomechanics of amyloid proteins and DNA nanostructures . . 35

4.4 4D structural dynamics of amyloid . . . . . . . . . . . 38

5 Conclusions and perspectives $\quad 41$

6 Acknowledgements $\quad 42$ 
7 Figure Captions

8 Bibliography 


\section{Introduction}

The development of innovative, inexpensive and sustainable approaches for energy conversion, the ability to process and store information produced at massive rates, and the understanding of the pathogenesis of human diseases, represent the main scientific challenges of the 21st century. In this scenario, nanometer-sized organic, inorganic and biological systems show great promises to satisfy these demands because of their appealing properties and functionalities, which stem from quantum confinement and pronounced surface effects. In recent years, this has led to the fabrication of increasingly complex nanoscale structures and the designing of advanced tools for their investigation, defining new frontiers in materials science, physics, chemistry and biology.

Nanomaterials have successfully been employed in a variety of applications. For instance, in semiconductors and strongly-correlated systems change of electronic and atomic structures with size and dimensionality allows one to manipulate their optical, thermal and electronic properties, which is crucial for the design of new generation optoelectronic [1], thermoelectric $[2,3]$, photovoltaic [4], and information storage [5] devices.

Similarly, the self-assembly of DNA molecules in nanostructures and nanomachines can open up the possibility of creating biomimetic and diseasetargeting units for both diagnostics and therapeutics [6]. Also, in biomaterials the modulation of the mechanical behavior at different length scales is critical for the biological processes that regulate the human body and the pathogenesis of diseases [7]. Despite such achievements, it is crucial to improve our understanding of fundamental processes at the nanoscale in order 
to further enhance the efficiency and performance of such devices.

Reduced size and dimensionality has profound effects not only on the ground state properties, but also on the dynamical behavior, which depends on the interaction between the different degrees of freedom and evolves though a series of nonequilibrium states. Electron density relaxations, phase transitions, atomic vibrations, and molecular rearrangements, which crucially determine the functionality of molecular assemblies and future nanodevices, generally occur on femtosecond $\left(10^{-15} s\right)$ to picosecond $\left(10^{-12} s\right)$ and nanosecond $\left(10^{-9} s\right)$ time scales. Time-resolved optical spectroscopies using visible, infrared and UV light in a pump-probe scheme are commonly used to study the dynamics in materials on ultrafast timescales, and can provide extremely valuable insights into their spatially-averaged dynamical behavior with a femtosecond temporal resolution.

However, at the nanoscale, non-averaged spatial information is required in order to fully understand and control the ultrafast processes, which play a key role for the future technological development. In this scenario, the static observation of matter is insufficient and only its visualization in both space and time, which requires the probing of the transient changes of materials with the appropriate temporal and spatial resolutions, can provide the fundamental knowledge for understanding its behavior. Moreover, we must be armed with techniques for single-particle imaging, which makes possible studies of heterogeneous systems and structural dynamics without demands for specimen crystallization.

Ultrashort electron pulses with energy ranging from few tens to few hundreds of keV exhibit a high scattering cross-section and a de Broglie wave- 
length on the order of picometers, allowing one to achieve high spatiotemporal resolutions in diffraction as well as in imaging and spectroscopy $[8,9,10]$. At Caltech, a novel technique, called four-dimensional electron microscopy (4D-EM) has been developed in the recent years $[9,11]$ where an ultrashort photon pulse is used for exciting the dynamics of interest and an ultrashort electron pulse is applied to probe the system as a function of the delay time between them.

For nanoscience, this approach has unique capabilities and significant advantages with respect to optical and X-ray probing: first, the possibility to reach a high spatial resolution (down to the atomic-scale), which cannot be obtained by optical techniques because of the long wavelength of the probe; second, the high sensitivity to small material volumes, such as monoatomic layers, which cannot be obtained using less-interacting X-ray photons; and third, the possibility to have a very compact table-top setup.

In this review, we guide the reader through particularly challenging problems, whose resolution could only be achieved when simultaneously reaching the limits of length and time scales involved. Our investigation spread from materials science to biology, providing the real-time access to electron and atomic dynamics on surfaces, at interfaces and in nanosystems, and gives an unprecedented insight into the non-equilibrium transformation pathways on their energy surface. After describing the basic concepts and the main characteristics associated with the different operation modes, several paradigm applications, characterized by a variety of spatial and time scales, are discussed. The numerous examples presented here will show the extreme versatility of the $4 \mathrm{D}$-EM technique and its capability in elucidating the dynamical 
behavior of advanced and complex nanomaterials.

\section{Basic concepts}

Fig. 1 shows a schematic of a typical 4D-EM setup. A pulsed laser delivers a train of optical pulses. A portion of the laser output is guided to the photocathode of the electron microscope and generates electron packets, which are used to probe the investigated sample. The dynamics of interest are initiated by the second output portion of the laser, which is delayed with respect to the electron probe using an optical delay stage. The electron beam after interaction with the sample is then recorded on a detection system allowing imaging, diffraction and spectroscopy $[12,13,14]$. This configuration, together with the different variants that will be discussed below, responds to the need for probing with high spatial and temporal resolutions the transient behavior of molecules, crystals and complex systems when excited in an outof-equilibrium state by an optical field, causing thermal heating or electronic excitation.

\subsection{Ultrafast diffraction}

Recent developments in ultrafast diffraction techniques have enabled the direct probing of the atomic-scale dynamics in several nanosystems using both transmission and reflection geometry. The transmission configuration is ideal for the investigation of free-standing thin layers $[15,16]$, single par-

ticles [17], nanotubes networks [18, 19], and biochemical specimens [20, 21], whereas the high surface sensitivity of the reflection geometry (grazing incidence) enables the observation of crystal surfaces [22], adsorbates [23], and epitaxial nanostructures $[24,25,26]$. 
Because nonequilibrium atomic motions lead to a change of the atomic displacement, and thus to a modification or loss of electron interference, the transient behavior of the diffraction pattern is able to mirror the lattice dynamics. Coherent lattice vibrations, thermal heating and phase transitions can cause the intensity of a particular diffraction peak to change. Moreover, the variation of the scattering vector for the observed Bragg reflections can elucidate a modification of the interatomic distances, thus allowing for the monitoring of homogeneous deformations (expansion/contraction) of the lattice [27].

With parallel-electron beam illumination, it is possible to accurately detect the longitudinal strain of the unit cell as driven by uniaxial stresses that compress or expand the lattice. When convergent-beam illumination is adopted, Kikuchi lines can be imaged in the diffraction pattern [28], and because Kikuchi diffraction is originated from multiple scattering of electrons in the material, the patterns are made by large cones of wave-vectors; Kikuchi lines are thus "attached" to the lattice planes and allow for a direct probing of their transverse (shear) dynamics [29, 30].

\subsection{Ultrafast microscopy of single particles}

In numerous applications and studies, real space imaging of single nanoparticles becomes crucial. Several methods have been implemented so far, and in the following we describe the ones most commonly used.

Using a tightly focused electron beam (convergent-beam geometry) in combination with the scanning capabilities typical of an electron microscope, it is possible to obtain a spot-by-spot mapping inside and outside the particle with nanometer resolution. When time resolved, this configuration enables 
the investigation of the nanoscale dynamics with sub-particle precision and femtosecond temporal resolution [31].

An alternative method to obtain spatiotemporal imaging of single, isolated nanoparticles adopts a parallel-beam illumination, rather than a convergent one. In this case, a small aperture is introduced in the image plane of the objective lens and only the particle of interest is isolated and detected [17]. In contrast with the convergent-beam mode, in this selected-area approach the electron beam is spread on a wide area, thus potentially preventing radiation damage effects, which is a fundamental aspect for the investigation of biological and organic materials.

Besides the two methods just described, conical-scanning dark-field imaging [32] can also be used to perform structural dynamics studies at a singleparticle level. In this method, the pulsed electron beam is tilted and conically scanned around the optical axis, so that the electrons diffracted by the sample can pass through a small aperture introduced in the back-focal plane and concentric with the optical axis. This approach is particularly effective when an ensemble of particles needs to be investigated. In fact, it allows for identifying the origin of all Bragg spots that form the diffraction pattern of the ensemble, thus providing a dark-field image of the corresponding single particles at each delay time.

\subsection{Ultrafast electron-spectroscopy}

By exploiting the energy-filtering capability of a transmission electron microscope, it is possible to perform electron energy loss spectroscopy (EELS), where the change in energy of the primary electron beam after interaction with the sample is measured. This method represents a powerful approach 
to investigate the electron density distribution and the electronic structure of materials [33]. When EELS is implemented in the 4D-EM setup described above, the collective dynamics of valence and core electrons can be obtained with femtosecond temporal resolution, enabling the spatiotemporal mapping of electronic structure changes [34, 18, 35].

While EELS is based on the inelastic interaction between the probing electrons and the valence/core electrons of the material, in the photon-induced near-field variant of energy-filtered 4D-EM, called Photon-Induced NearField Electron Microscopy (PINEM) [36], an optical pulse is synchronous with the electron pulse and the energy exchange takes place between photons, electrons and the sample. As described in details below, the PINEM configuration allows one to map the transient behavior of the near-fields created by the photons in the vicinity of a nanostructure, and represents an innovative imaging method for nanoscale materials science and biological systems.

\subsection{Scanning ultrafast microscopy}

For the investigation of real-space dynamics in material surfaces and interfaces, transmission geometry is not suitable and scanning electron microscopy (SEM), which provides the unique capability of obtaining three-dimensionallike images, has to be adopted. Ultrafast SEM [37, 38] shares with the transmission configuration a similar scheme of photon-pump/electron-probe, although exhibits a different detection setup. An ultrashort electron packet is photogenerated by the sharp field-emitter tip of the SEM microscope, and pixel-by-pixel recording of secondary electrons and primary backscattered electrons takes place instead of a parallel processing of the image. 
In the scanning configuration, thick specimens can be examined with a lateral resolution of few nanometers. This provides several advantages with respect to the transmission configuration, such as minimization of the effort for sample preparation, reduction of radiation damage from laser and/or electrons, and the presence of a heat sink for temperature-jump experiments. Moreover, because the escape length of secondary electrons produced during electron/sample interaction is only a few monolayers, the method has a high surface sensitivity, opening up investigations of reactive surfaces, molecules and biological materials.

\section{Applications: Materials Science}

The potential of nanomaterials for energy conversion and data storage applications has motivated scientists to investigate these materials under nonequilibrium operating conditions. Four-dimensional visualization of matter, in space and time, plays a fundamental and unique role in the study of mechanisms, and hopefully in the design and engineering of advanced applications. In particular, observing the motion of atoms and electrons as excited by an eternal stimulus can provide a crucial understanding of the non-equilibrium properties of these materials, as described below in particularly paradigm cases.

It is worth mentioning that for all applications where high spatial, temporal, and energy resolutions are needed, single-electron packets (or pulses containing at most few tens of electrons) are employed. Under this condition, Coulomb repulsion within the "electron bunch" can be circumvented, and broadening in space, time and energy becomes absent, although a small 
contribution can exist from the initial kinetic energy spread [39].

\subsection{Nanoscale acoustic phonon dynamics}

Understanding and controlling propagation of sound and heat, described as atomic vibrations transmitted through the lattice (acoustic phonons), may provide unprecedented opportunities for new and broad technological developments, reaching what has been described in a recent review [2] as a phononic technological revolution. For energy conversion, transport and localization in advanced thermoelectric, heat management and acousto-optical applications, one needs to be able to accurately manipulate hypersound (frequency, $f \sim 1-100 \mathrm{GHz})$ and heat $(f \sim 0.1-100 \mathrm{THz})$. This requires the design, fabrication and characterization of composite materials whose length scale ranges from several hundred to few nanometers. In this scenario, direct observation of atomic motions becomes crucial and requires the probing of the transient changes of the system with ultrafast temporal and atomic spatial resolutions, which can be achieved by adopting ultrafast electron diffraction and imaging methods.

A striking demonstration of the ability of time-resolved diffraction to map the anisotropic structural dynamics of nanomaterials at the atomic scale is given by several recent works on silicon waveguides, graphite nanofilms and GaAs quantum dots, as discussed below.

Using time-resolved Kikuchi diffraction, in convergent-beam geometry, Yurtsever and Zewail [29] studied the dynamical behavior of a 120-nm thick acoustic waveguide, made of a wedge-shaped silicon single crystal, when illuminated by a femtosecond laser. The authors observed resonance oscillations of the unit cells with frequency of $33 \mathrm{GHz}$ and amplitude of $0.3 \mathrm{mrad}$ 
(corresponding to a total lateral movement of the atomic layers of $0.2 \AA$, well below the interatomic distances). These oscillations have the form of a Gaussian-like wave-packet envelope propagating along the direction normal to the wedge. From the wealth of information provided by the Kikuchi pattern, the amplitude, phase and polarization of the coherent lattice vibrations were characterized and allowed to elucidate the origin of this behavior, which is associated to the coupling between longitudinal and transverse waves in the case of a wedge geometry. In fact, the laser-driven thermal expansion of the silicon slab creates a compression/expansion along the longitudinal direction, resulting in the so-called stationary breathing modes of the slab. Because silicon has a finite Poisson ratio and for a wedge geometry it exists a gradient in the thickness of the slab, the longitudinal motion couples with a non-stationary "transit-like" transverse motion, [40] which then creates the observed wave-packet.

From the case of the silicon waveguide just described, we have learned that coupling between longitudinal and transverse lattice vibrations can occur in presence of the breaking of the translational symmetry (the wedge) within the lattice. However, this is not limited to artificially-made structures, but can in principle exist as well in materials with anisotropic thermal expansion when laterally constrained, such as a graphite nanocrystals. Liang et al. [30] used ultrafast optical pulses to excite lattice vibrations in a 200-nm thick graphite sample and recorded the time evolution of the Kikuchi pattern. The results revealed strong vibrational features at 9.1 and $75.5 \mathrm{GHz}$ with amplitudes of 0.1-0.2 mrad (corresponding to a total lateral movement of the atomic layers of 0.1-0.2 $\AA$ ), as evidenced by Fourier transforms of 
Kikuchi line evolutions. The 9.1-GHz wave is representative of the longitudinal out-of-plane motion of the graphene planes along the stacking direction (c-axis), while the $75.5-\mathrm{GHz}$ mode is associated to a shear in-plane wave originated from the coupling between longitudinal and transverse vibrations within the laterally constrained graphite nanocrystal. The ability to access the transverse strain dynamics and associated lattice deformations can give significant insights into the strain-stress relationship of materials. In fact, for graphite the authors found that this $75.5-\mathrm{GHz}$ mode exhibits characteristic polarization properties and a long lifetime (milliseconds) compatible with a localized breather motion, which represents the nonlinear elastic response of the lattice to the ultrafast excitation.

So far we have discussed the dynamics of acoustic waves in hundreds-nm large nanostructures, which can be described within the framework of general theory of elasticity. However, when the length scale of the investigated structures becomes of the order of several nanometers, such as in low-dimensional systems, the propagation of atomic vibrations is strongly altered. By directly monitoring the atomic motions after fs optical excitation in GaAs quantum dots, Vanacore et al. [26], using ultrafast electron diffraction in reflection geometry (Fig. 2a), were able to visualize, for the first time, the coherent "ballistic" behavior of acoustic phonons when they travel within the quantum dots. The authors monitored the time constants of the anharmonic phononphonon decay channels, and their dependence on the dot size, excitation fluence and scattering angle (Fig. 2b, 2c-left and 2d-left). As a comparison, in bulk GaAs, optically excited electrons couple in $\sim 100$ fs with longitudinal optical (LO) phonons, which then decay toward high-energy acoustic 
phonons in $\sim 3.5$ ps. Further decay toward low-energy (thermal) modes is then reached in tens of ps. For GaAs dots with dimensions smaller than the inelastic phonon mean-free path $(\sim 26-27 \mathrm{~nm})$, the authors found a decay constant of $\sim 3.7 \mathrm{ps}$, thus deducing that the excitation of thermal motions is quenched and the energy remains localized in high-frequency $(f \sim 4 T H z)$ acoustic modes that travel within the dot without interacting with other phonons (no collisions, ballistic transport). In this case, the lattice preserves its structural coherence and, a breathing oscillation of the interatomic distance with amplitudes of $\sim 2 \mathrm{pm}$ (well below the lattice constant of GaAs) is clearly visible (Fig. 2c-right). As the dot size increases, the observed decay constant becomes $\sim 12.5 \mathrm{ps}$, suggesting that phonon-phonon scattering within the acoustic population now takes place, and the energy transport becomes diffusive in nature, similar to what happens in the bulk case. This process induces a loss of coherence in the structural dynamics and, hence, the absence of the breathing oscillations (see Fig. 2d-right). These results are fundamental for the understanding and control of energy-conversion in nanoscale materials suitable for thermoelectric applications.

Phonon management is a newly emerging field, rapidly developing with real potential for innovations in new applications of materials and devices. Recent technological advances have opened up new opportunities for the integration of graphene and other 2D crystals, such as monolayers of transition metal dichalcogenides, in energy-conversion applications [41]. In this context, the ability to optically control the amplitude of the atomic vibrations, using for instance phase-locked two-pulse excitation or intense $\mathrm{THz}$ optical fields, coupled to the possibility of visualizing the energy transport at the atomic- 
scale as described in the previous studies, would provide a key understanding on how to suppress or enhance thermal and electrical conductivities in $2 \mathrm{D}$ materials.

\subsection{Nanoplasmonics and Nanophotonics}

Observing and controlling localized electric fields created within or in the vicinity of nanostructures is of fundamental importance in photonic and plasmonic applications. Near-field evanescent waves can effectively excite plasmonic resonances, thus the attraction for exploiting their large intensity, propagation and polarization for efficient photovoltaic operation, lightharvesting, and biological sensing [42]. In the far-field, photonics evolve, and now with unique space and time characteristics [44].

Several methods have been used to probe optically-induced electric fields. The most common approach is represented by tip-enhanced near-field optical microscopies, which can measure evanescent electric fields with subwavelength spatial resolution ((sub)hundred nanometers) [43]. Despite its large use, this method exhibits several drawbacks, such as the low signal intensity, the slow point-by-point acquisition, the influence of the scanning tip, and the difficulty of probing "buried fields". All these issues can be overcome by using a specific variant of energy-filtered 4D-EM, called Photon Induced Near-field Electron Microscopy, PINEM [36, 45], which provides higher spatial resolution, together with the versatility of imaging either single nanostructures using a scanning convergent-beam or many particles with parallel acquisition. When ultrashort electron pulses are synchronized with fs optical pulses in the energy-filtered 4D-EM setup shown in Fig. 1b, the high spatial and energetic resolutions typical of electron and optical microscopies, respectively, can be 
combined with the ultrafast temporal resolution to map in space and time the evanescent near-fields created in the vicinity of a nanostructure.

In free space, electrons and photons do not interact with each other because of momentum mismatch. The momentum exchanged by an electron during an electron-photon scattering event is $\Delta p_{e}=\hbar \omega / v$, where $\hbar \omega$ is the photon energy and $v$ is the electron speed. For electrons at $200 \mathrm{keV}, v$ is $0.7 c$, where $c$ is the speed of light. Because the momentum of a single photon is $\Delta p_{p h}=\hbar \omega / c$, smaller than $\Delta p_{e}$, the momentum cannot be conserved and electrons and photons cannot couple. However, in presence of a nanoparticle, the spatially confined near-field created by surface plasmon excitation, can provide the necessary momentum contribution along the electrons propagation direction such that inelastic scattering can take place. This is dictated by the uncertainty principle $(\Delta z \Delta p \sim h)$, which requires a spatial confinement of $\Delta z \approx 2 \pi v / \omega$ in order to satisfy the momentum conservation. For photons with $\hbar \omega=2.4 \mathrm{eV}$ and electrons with $v=0.7 c, \Delta z$ is around 350 $\mathrm{nm}$, where $z$ is the propagation direction. It follows that a nanoparticle with size smaller than this value will generate a scattering potential with a significant momentum contribution such that inelastic coupling between ultrafast electrons and the electric field effectively occurs. The discovery and the applications, together with the theoretical developments [46], have began at Caltech and the unraveling of nano-scale phenomena is continuing, the latest of which is "photon gating" [47].

Yurtsever and Zewail [44] have introduced the PINEM technique to visualize the optically-induced near-fields in the vicinity of silver spherical nanoparticles, and have studied their dependence on the particle size, shape 
and separation. As schematically shown in Fig. 3a, a particle with size $d$ is excited by an ultrafast optical pulse with wavelength $\lambda(\lambda \gg d)$, creating an evanescent electric field in its vicinities [48]. An ultrashort electron pulse, synchronized with the photon pulse, is inelastically scattered by the longitudinal component of the near-field and a quantized energy exchange occurs, generating well-resolved gain and loss peaks in the electron energy spectrum [36]. By selecting only the energy-gained electrons (1-10 eV) to form an image, the evanescent field created in the vicinity of the particle can be visualized, as shown in Fig. 3b. Since the particle size is much smaller than the wavelength of light, its dipolar response and dipole-dipole interaction are the dominant features in the PINEM images. This is also evident in the images of Figs. 3c and 3d where the orientation of the induced dipole follows the rotation of the polarization of the excitation light. Very interestingly, an entanglement effect between two particles appears when their separation is comparable or smaller than the decay length of the evanescent fields [49]. In this condition, a coherent sum of the near-fields takes place (interference), generating void-field channels that connect the two particles between the points on their surfaces where the electric field vanishes (see Fig. $3 \mathrm{e})$. Because of the dipolar origin of these channels, their size and orientation can also be controlled by the polarization of the incident field, particle size and separation.

Besides the ability to visualize the near-field generated outside the investigated nanoparticle, when adopting a tightly focused electron beam scanned across the optically-excited nanostructure, PINEM can also provide imaging of the field distribution inside the particle. In an elegant proof-of-principle 
experiment, Yurtsever et al. [31] have adopted this approach on a single triangular silver nanoparticle, and were able to reconstruct energy-space-time maps of the transient field. Fig. 3f shows the intensity of the PINEM signal obtained by selecting the first energy-gain peak $(+1 h \nu)$, when complete synchronization between optical and electron pulses occurs $(t=0)$. The mapping reveals that the strongest field intensity is localized at the right vertex and at the left edge of the triangular particle. Because the particle has an edge length of $130 \mathrm{~nm}$, smaller than the excitation wavelength of 518 $\mathrm{nm}$, the charge density within the nanoparticle would exhibit a dipole-like behavior following the linear polarization of the excitation light, similar to what happens for the spherical particles described above. However, for a triangular shape, the charge accumulated on the flat edge is pushed toward the vertices by Coulomb repulsion. This charge redistribution is thus responsible for the observed spatial distribution of the evanescent near-field.

From these examples it is clear that PINEM represents an ideal tool for the investigation of optically-induced electric fields in photonic circuits used as building blocks in nanoscale plasmonic devices $[45,50]$. Because the temporal and spatial coherence of the generated near-field strongly affect the performance of these devices, it is extremely important to reach a precise control of these characteristics. This could be achieved by exploiting, within the PINEM setup, the constructive or destructive interference between electron populations excited using two phase-locked optical pulses with different wavelength, e.g. the first and second harmonics. As discussed in a previous work [46], preliminary results indicate that energy gain and loss can be selectively controlled by adjusting the phase difference between two optical pulses 
with wavelength of 1038 and $519 \mathrm{~nm}$. Therefore, we could expect that, by tuning the phase difference between the two optical pulses, coherent control of the spatial extent or the temporal duration of the created near-field could be obtained.

\subsection{Nanofriction}

Phenomena involving the atomic interaction at the interface between two different materials, such as friction, lubrication and wear, are ubiquitous in nature, and play a fundamental role in many technological applications [51]. When reaching the nanoscale, the main challenge is represented by the possibility to directly visualize the motion at the interface with sufficient time resolution to capture the fast nonequilibrium dynamics.

Using 4D-EM, Flannigan et al. [52] investigated the solid-solid friction forces at the interface between a single crystal of copper 7,7,8,8-tetra-cyanoquinodimethane, $\mathrm{Cu}(\mathrm{TCNQ})$, and an amorphous membrane (substrate). The motion of the crystal is obtained by the expansion induced by a pulsed laser excitation, and the friction forces are determined by monitoring the displacement of two crystal surfaces, the one in direct contact with the substrate and the opposite one exposed to the vacuum. From the measured lag time (6 ns) and the top edge displacement (3 nm) between these two surfaces, a static friction force of $0.5 \mu N$ was obtained using the Hookes's law $(F=-k x)$. By studying the restoring motion of the surface in contact with the substrate, a rate of steady-sliding of $0.85 \mathrm{~nm} / \mu$ s was measured. From this value and applying again Hooke's law in hypothesis that the crystal moves at a fixed velocity, a dynamic friction of $0.4 \mu N$ was derived. For the case of the investigated metal-organic crystals, the force of gravity is on the order of 
piconewtons and therefore cannot explain the measured values for the static and dynamic frictions. However, considering chemical interactions at the interface, these can be attributed to the Coulombic/van der Waals adhesion forces between the crystal and the substrate [53], which are then expected to be on the order of micronewton, i.e. 6 orders of magnitude larger than the gravity.

In addition to this smooth sliding, the authors were also able to visualize a periodic slip-stick behavior (see Fig. 4a). This process involves an abrupt detachment of the crystal from the substrate, creating a fast slip motion, followed by a rapid reattachment that keeps the crystal blocked on the substrate for a longer time (sticking event). This results in a sawtooth dynamics for the friction force with a periodicity of $45 \mathrm{~ns}$ and slip-stick transitions with an average time constant of $2.8 \mathrm{~ns}$. The work by Flannigan et al. [52] thus demonstrates for the first time that only when invoking suitable temporal and spatial resolutions, the nonequilibrium friction between two materials generated by an impulsive laser excitation can be visualized.

Besides solid-solid interactions, a key aspect in nanofriction is represented by the investigation of viscous friction at a solid-liquid interface, especially when liquids are confined in nanometer-wide environments. To tackle this issue, Lorenz and Zewail [54] employed a new approach, where, first, the lead core of a $\mathrm{ZnO}$ nanotube is melt by heating with a single laser pulse, and then, using single-shot 4D-EM, the molten lead is imaged while flowing within the nanochannel. As shown in Fig. 4b-d, after the laser heating, the lead column partially filling the tube rapidly expands, with a nearly constant velocity of $3.9 \mathrm{~m} / \mathrm{s}$ in the first $30 \mathrm{~ns}$. After reaching the maximum expansion 
in about $60 \mathrm{~ns}$, a slower shrinking toward its initial length is then observed. By comparing the initial expansion behavior with the results of an analytical model used to describe the fluid motion, the authors were able to obtain a quantitative estimation of the viscous friction coefficient, which turns out to be only $6 \%$ of the value for macroscopic continuum dynamics. This has a direct implication on the shape of the velocity profile for the moving liquid column within the tube, which becomes almost flat as mediated by the strong interaction between the liquid lead and the $\mathrm{ZnO}$ nanotube walls.

The possibility of using the approach of Lorenz and Zewail [54] for the investigation, with high spatial and temporal resolutions, of nanofluidic structures and biological channels, such as water confined in carbon nanotubes or between graphene sheets, represents the natural extension of this work in the near future.

\subsection{Phase transitions}

Complex systems, such as macromolecular structures or strongly-correlated solids, involve a multidimensional energy landscape with multiple transition states, which crucially depend on the length scale involved and on interaction with the external environment. Understanding the transformation pathway among the different phases, with the suitable spatial and temporal resolutions, is essential for elucidating the underlying physics and controlling the ultimate performance of new applications [8, 55].

As described by Baum et al. [56] in the case of vanadium dioxide $\left(\mathrm{VO}_{2}\right)$, a prototypical electron-correlated system, ultrafast electron diffraction is used to visualize the transitional lattice structures during the insulator(monoclinic)to-metal(tetragonal) transformation. Photoexcitation primarily involves bond- 
ing electrons of the vanadium pairs, which are responsible for the stabilization of the initial monoclinic structure. The excitation to antibonding states results in a repulsive force, which moves the vanadium atoms in the $\mathrm{V}-\mathrm{V}$ pairs apart from one another on a fs time scale. As mediated by electron-phonon coupling, long range displacement of atoms within the unit cell takes place on a ps time scale, and only at later times (hundreds of ps) acoustic shear waves drive the lattice toward the final tetragonal structure. These observations indicate that stepwise atomic motions, rather than a direct structural conversion, mediates the phase transition in $\mathrm{VO}_{2}$, as also reported by Morrison et al. [57].

An alternative perspective of the same transformation has been recently provided by Hassan et al. [47], who have probed the dielectric response of the $\mathrm{VO}_{2}$ crystal during the insulator-to-metal transition. They used a modified version of the PINEM method, where energy-gain electrons generated by an optical pulse, synchronous with the electron pulse, are used to probe the dynamics initiated by a second optical pulse. From the measured dynamics of PINEM intensity, as compared with ultrafast diffraction data, the authors were able to retrieve the change of the dielectric constant associated with the stepwise atomic motions of the transition, opening up the possibility of studying phase transformations from an electronic, instead of a structural, point of view.

The distinct pathways evidenced for the phase transition in $\mathrm{VO}_{2}$ are not peculiar to this particular case, but are indicative of a general behavior. In fact, similar elementary steps have been shown to define the structural pathways and the nonconcerted nature of the crystalline-to-amorphous transfor- 
mation in GeTe [58], and of the amorphous-to-crystalline transition in $\mathrm{TiO}_{2}$ [59]. For GeTe, a prototypical phase-change material used in data storage devices, a non-thermal solid-solid transformation from the initial rhombohedral phase toward an intermediate cubic structure is found to occur in $12 \mathrm{ps}$, before reaching the amorphous state in hundreds of ps. The cubic phase acts as a precursor for the final amorphous structure and can potentially limit the maximum speed of data recording. Similarly, in $\mathrm{TiO}_{2}$ the crystallization pathway from the liquid state, which is particularly relevant for energy-conversion and photocatalysis applications, exhibits a unique twostep dynamics. The crystallized fraction of material exhibits a robust plateau extending over a microsecond, which reflects the presence of intermediate state(s) before reaching the final crystal ordering.

Reduced size and dimensionality, when compared to the case of bulk crystals or nanofilms extending over dimensions of several micrometers, as those described above, can strongly modify the dynamical pathway of the phase transitions. Moreover, for the realization of new generation devices that exhibit a characteristic length scale on the order of nanometers, the ability to observe the spatiotemporal behavior of individual nanostructures, as compared with the dynamics of an ensemble, becomes essential to unveil the nature of the transformations governing their physical and chemical properties. In a recent publication, van der Veen et al. [17] reported the visualization of the structural details during an electronic phase transition in a single, isolated nanoparticle. The experiment was conducted in $\mathrm{Fe}($ pyrazine $) \mathrm{Pt}(\mathrm{CN})_{4}$ metal-organic nanocrystals, which, under laser heating, undergo a cooperative transition from a low-spin (LS) to a high-spin (HS) state. As shown 
by both real space imaging and reciprocal space dynamics (Fig. 5), after laser excitation a highly anisotropic expansion (in both space and time) is observed. This is expected for the LS $\rightarrow$ HS transition, where an initially strained, buckled particle in the LS state releases the stress and reaches a structurally relaxed HS state [60]. The transition from a strained to a relaxed state can be also clearly seen in the diffraction contrast present within the images in Fig. 5a. The initially localized scattered dark contrast spreads under the laser excitation and almost spans the entire nanocrystal at a delay time of $50 \mathrm{~ns}$, partially reforming only at later times.

The versatility of $4 \mathrm{D}-\mathrm{EM}$ allowed the authors to investigate also the dynamics of an ensemble of $\mathrm{Fe}($ pyrazine $) \mathrm{Pt}(\mathrm{CN})_{4}$ particles as compared to the evolution of a single one. For a single particle, where the contact area with the substrate is very high, the LS $\rightarrow$ HS transition is very fast. On the contrary, for a heterogeneous ensemble of particles, the significantly reduced contact area with the substrate becomes responsible for a slower LS $\rightarrow$ HS transition. This clearly shows how the interaction with the external environment can affect the phase transformation and ultimately control the performances of the material.

These results represent the first step toward the understanding of spinswitching processes in molecular memories and data storage devices that employ metal-organic nanoparticles. The next frontier will be the exploration of phase transformations in heterogeneous systems, such as solid-liquid or solid-gas interfaces, providing crucial information on the dynamic behavior in conditions that are closer to synthesis and device operation. 


\subsection{Carrier transport}

Carrier excitation, localization and transport play a fundamental role in a wide range of physical phenomena that crucially affect the operation of photovoltaic, thermoelectric, photonic, and quantum computing devices [61]. Although carrier dynamics has been extensively studied in bulk materials, a substantial lack of relevant experimental data and an incomplete understanding of the microscopic transport details is the case for nanostructures, surfaces and interfaces, where the reduced dimensionality and the higher surface-to-volume ratio can significantly alter these processes. The challenge lies in the difficulty of simultaneously reaching the limits of length and time scales involved. Scanning ultrafast electron microscopy (S-UEM) [37], which enables simultaneous ultrashort temporal and nanometer spatial resolutions, exhibits a high sensitivity to valence and conduction electrons at the sample surface, making this technique the ideal tool for visualizing nanoscale carrier dynamics in both space and time.

In a recent publication, Najafi et al. [62] reported the direct imaging of carrier interfacial dynamics in a silicon p-n junction. As schematically depicted in Fig. 6a, transport toward the junction of electron and holes excited by the optical pump is imaged by scanning the pulsed electron probe on the area of interest and detecting secondary electrons (SEs) at each position as a function of the time delay. The experimentally-measured temporal behavior of charge carriers at the surface of the diode is shown in Fig. 6b. After the fs optical excitation, electrons are promoted to the conduction band in both n-type and p-type regions, which acquire a bright contrast because of the larger electron density available in high energy states (frame at $+6.7 \mathrm{ps}$ ). 
On the contrary, the depletion region remains dark because of the built-in electric field that hinders SE detection. On a time scale of several tens of ps $(+36.7-80 \mathrm{ps})$, charges are transported toward the junction. Here, the minority carriers are able to cross the junction whereas the majority carriers are reflected away (gating effect), inducing a net charge separation that results in excess electron density (bright contrast) and hole density (dark contrast) in the n-type and p-type regions, respectively. Finally, at longer delay times $(\sim 1 \mathrm{~ns})$, relaxation toward the equilibrium takes place as carriers move back across the junction to recombine.

A very interesting aspect of the measured behavior is that the observed charge separation occurs over a spatial extent of several tens of micrometers within the first $\sim 80 \mathrm{ps}$ (rate of $\leq 1 \mu \mathrm{m} / \mathrm{ps}$ ). This rapid transport is not expected within the framework of the drift-diffusion model [63], according to which carrier drift occurs only within the depletion region (few microns wide in this case) with a diffusion coefficient as low as $10-30 \mathrm{~cm}^{2} / \mathrm{s}$. As theoretically described by the authors (see inset in Fig. 6a), this behavior could only be explained by considering a ballistic-type motion, where carriers with a very high excess energy, equivalent to a temperature of $10^{4} \mathrm{~K}$, move on the surface at a relatively high speed, on the order of $10^{6} \mathrm{~m} / \mathrm{s}$, without suffering significant quenching by the interaction with lattice phonons.

The ability to visualize the microscopic details of the carrier transport as described by Najafi et al. [62] paves the way for further fundamental studies of charge dynamics in nanoscale structures and organic materials, in configurations similar to those used in a real device, such that predictive information for future design can be obtained. Moreover, the manipulation of 
the spatial and temporal characteristics of the applied electromagnetic wave, initiating the dynamics, can also open up the possibility of controlling, and not only observing, such transport down to the electron dynamics time scale and the nanometer spatial scale.

Other novel studies from this laboratory include the investigation of GaAs surfaces [64] and graphene layers [65]. In GaAs the authors showed that the modification of the surface electronic structure obtained by modulating the crystal doping and the carrier concentration has profound effects on the electron/hole dynamics and carrier-carrier scattering. In graphene, crater-like spatial profiles of the surface carrier distribution were observed at high excitation fluences, and accounted for by an inverse Auger mechanism occurring when a defined excitation threshold is reached.

\subsection{Valence and core-level excitation}

The dynamical evolution of the electron density and its interaction with other degrees of freedom, such as lattice, orbital and spin, are at the origin of a variety of phenomena in the condensed phase, and they are fundamental for transport, optical and magnetic properties. When Electron energy-loss spectroscopy (EELS) is implemented in the 4D-EM setup described above, the dynamics of the electron distribution can be probed with ultrafast temporal and nanometer spatial resolutions.

The low-energy region $(0-50 \mathrm{eV})$ of the EEL spectrum is characterized by valence band transitions and plasmon excitations, rendering it sensitive to changes of the overall electron density of the material. Because of the peculiar electronic and atomic structures, carbon nanomaterials are considered as prototypical systems to investigate electron-lattice interaction at the 
nanoscale. Carbone et al. [34] and Vanacore et al. [18] have monitored in graphite and carbon nanotubes, respectively, the transient behavior of the $\pi$ and $\pi+\sigma$ plasmon bands following fs optical excitation, and compared it to time-resolved diffraction data. The approach used in these works allowed to investigate the ultrafast nonequilibrium distortion of the lattice as induced by the occupation of antibonding $\pi^{*}$ orbitals. Particularly interesting is the case of graphite, where the structural deformation is associated to a change of the electronic hybridization from $s p^{2}$ (2D, graphene) to $s p^{3}$ (3D, diamond) [34].

A significant advancement in time-resolved EELS would be the possibility of probing the dynamics of core-level electron excitations, which characterize the high-energy region $(>100 \mathrm{eV}$ ) of the EEL spectrum. In fact, this would provide the unique opportunity to observe the transient change of the $l o$ cal electronic and atomic structures surrounding the absorbing atom, which cannot be accessed by electron diffraction and low-loss spectroscopy.

In a recent publication, van der Veen et al. [35] have implemented ultrafast core-loss spectroscopy in 4D-EM, and applied it to the study of photoexcited graphite (see also Ref. [66]). The authors probed the carbon $K$ edge spectrum at $\sim 280-450 \mathrm{eV}$ following ns and fs optical excitations. By comparing the experimentally-measured transients with molecular dynamics calculations, they were able to identify a local increase of the $\mathrm{C}-\mathrm{C}$ bond length within the $a b$-planes of graphite, although the overall behavior of the lattice is still represented by an in-plane contraction. These results highlight the important role played by the anharmonicity of the interatomic potential, responsible for the local C-C bond length increase, and out-of-plane phonons, 
responsible for the long-range in-plane contraction [67], in the overall lattice dynamics of graphene and graphite.

In addition to local structural information, time-resolved core-level EELS also provides insights into the dynamics of high-energy electronic states, otherwise unaccessible by low-loss electron microscopy and optical spectroscopies. In graphite, van der Veen et al. [35] observed a transient red shift on a ps time scale of the $\mathrm{C}$ peak at $290 \mathrm{eV}$, which is associated to transitions of core electrons into empty $\sigma^{*}$ states. The authors explain this behavior as due to an ultrafast shrinkage of the band gap between filled $\pi / \sigma$ - and empty $\pi^{*} / \sigma^{*}$-bands. Considering that on the ultrafast time scale a considerable amount of energy is deposited in optical and acoustic phonons, the major contribution to the gap shrinkage would be determined by the direct renormalization of band energies due to electron-phonon interactions as theorethically described by Olguin et al. [68]. Because of the local sensitivity of core-loss spectroscopy, numerous future applications in the study of nanoscale chemical dynamics, charge transfer and phase transitions are foreseen.

\section{Applications: Biology}

In order to fully understand the molecular mechanisms behind biological processes, the investigation of the static structure of the proteins and DNA systems involved has become an important part of the narrative; the other part is dynamics! A dynamical approach on a time scale ranging from the femtoseconds of nuclear motions to nano and microseconds needs to be adopted. In the following, we report several biological applications of four- 
dimensional 4D electron microscopy, where (ultra)fast imaging, diffraction and spectroscopy are used to provide the real-time dynamics of protein vesicles, bacteria, amyloid proteins, and DNA nanostructures.

\subsection{PINEM imaging of biological specimens}

Nowadays, a continuous and increasing effort has being devoted to the development of imaging techniques able to visualize biological materials with high spatial resolution such that their inner microscopic structure can be revealed. Recent optical methods based on super-resolved fluorescence microscopy [69] (see also the 2014 Nobel prize in Chemistry work) have pushed the spatial resolution below the diffraction limit and allowed for the study of proteins and cells in-vivo. Although very powerful, these optical methods are based on fluorescence emitters and cannot reach the atomic sensitivity typical of electron microscopy, as exquisitely demonstrated in the structural mapping of the ribosome [70]. However, in standard TEM the obtained images are static, i.e. averaged over seconds of acquisition time, and suffer of inherently weak contrast because of the low-Z (Z, atomic number) elemental composition of biological materials and the need for thin specimens.

As described above, when ultrashort electron pulses are synchronized with fs optical pulses in the presence of a nanostructure, the high spatial and energy resolutions typical of electron and optical microscopies can be combined with the ultrashort temporal resolution, enabling the mapping in space and time of the evanescent near-fields created in the vicinity of the nanostructure [36]. Because intense near-fields are produced at the edge and at the sur-

face of the observed structures, this method creates an image with enhanced contrast, while simultaneously providing ultrashort temporal and nanometer 
spatial resolutions.

This imaging has been experimentally demonstrated in biological materials by Flannigan et al. [71], who have performed PINEM imaging of protein vesicles and whole cells of Escherichia coli. The vesicles are composed of a dielectric shell of BSA protein molecules encapsulating a liquid core. When only the electrons that have gained energy in the interaction with the photons are selected, a PINEM image of a protein vesicle can be obtained (see Fig. 7a-right), showing that at the edge of the vesicle, which is the region where the protein resides, exists an enhanced contrast with respect to the rest of the field of view. As compared with a brigh-field TEM image of the vesicles shown in Fig. 7a-left, the intensity created by the surrounding substrate, that lowers the contrast at the edge of the vesicle in the standard TEM mode, is absent in the PINEM image. The spatial location of the contrast enhancement can be also controlled by changing the orientation of the polarization of the fs laser (see Fig. 7b). The power of the method and the significance of this effect become evident when investigating a biological structure with increased complexity with respect to simple protein vesicles, such as an unfixed and unstained cell of the Escherichia coli bacterium. Comparing the image of the E. coli cell acquired in PINEM mode (Fig. 7c-right) with that acquired in bright-field TEM mode (Fig. 7c-left), an enhanced contrast is observed at both the outer and inner regions of the cell, making visible intracellular topological features otherwise not detectable.

The advances in the visualization of biological materials provided by the PINEM approach, coupled with the possibility to use environmental cells, would allow one to perform high resolution imaging of cells and proteins in 
liquids. The spatial resolution is ultimately determined by the decay in space of the field of the nanostructure. In addition, the introduction of a second optical pulse to the experimental setup would initiate the material dynamics [47] and enable investigation of their transient behavior under an external stimulus.

\section{2. $4 D$ cryo-electron microscopy}

In recent years, the investigation of proteins and cellular structures in their native-like conditions has been carried out mainly using the cryo-electron microscopy technique. In this method, the sample contained in water is quickly plunged into liquid ethane, freezing the water into a glassy (vitreous) ice that does not perturb the image or disrupt the sample as ordinary crystalline ice would do.

Judging from the amount of high quality works $[72,73,74,70,75,76]$ being performed worldwide, cryo-EM can be definitely considered as the future of structural biology. In this regard, the introduction of the time dimension into cryo-EM would permit the visualization of the dynamic behavior of biomolecules in a fully hydrate state. This has been experimentally demonstrated by Fitzpatrick et al. [77], who monitored the dynamics of a thin film of photoresponsive insulin amyloid fibrils embedded in vitreous ice (Fig. 8a-b) when excited with a precisely timed laser pulse. Amyloid proteins are polypeptide aggregates formed by hydrogen-bonded $\beta$-sheets running parallel to the long-axis of the fibril [78]. Efficient heat transfer to the fibrils was obtained by binding a small amyloidophilic dye molecule, Congo red, to their outer surface.

By monitoring the characteristic reflection related to the regular inter- 
strand spacing of $0.48 \mathrm{~nm}$ within the $\beta$-sheets (Fig. 9b, lower panel), the authors were able to track the stretching of the unit cell along the cross- $\beta$ interface upon a laser-induced temperature jump from an initial temperature of $118 K$. A relative expansion of $11 \pm 1.2 \times 10^{-3}$ (Fig. 8c-left) was measured, corresponding to a movement of the $\beta$-strands of $\left(11 \times 10^{-3} \times 0.48 \mathrm{~nm}\right) / 2=2.7 \mathrm{pm}$ in opposite directions (Fig. 8c-right). This value is almost twice as large than that measured in the same experimental conditions for fibrils kept at the same initial temperature of $118 K$ but in absence of glassy ice. Since amyloid fibrils are stabilized by a network of interbackbone hydrogen bonds [78], the observed increased expansion is therefore indicative of a weakening of this hydrogen-bonding network in presence of water, making the fibrils more stretchable in the aqueous environment.

The approach adopted by Fitzpatrick et al. [77] is a clear step forward in the field of dynamical biology, and paves the way for the time-resolved investigation of conformational changes in near-native condition of protein crystals with increased complexity, such as 2D membranes protein crystals and 3D micro- or nano-crystals embedded in vitreous ice.

\subsection{Biomechanics of amyloid proteins and DNA nanostructures}

Knowledge of the degree of stiffness and robustness of materials is critical

to the understanding of their mechanical stability, energy absorption/dissipation, and failure under stress. In biological systems, all these properties determine their pathological and molecular functions, actively influencing the behavior of those structures with which they interact and ultimately affecting the pathogenesis of human disorders and diseases.

The current methods used for the determination of the rigidity, or Young 
modulus, $Y$, of biological materials, such as statistical analysis of shape fluctuations, single molecule stretching, and atomic force microscopy (AFM) nanoindentation, usually provide conflicting results and display a large range for the measured $Y$ values. This is particularly evident for amyloid proteins, which are associated with more than 50 human disorders (from Alzheimer's disease to type II diabetes [79]). In this case, $Y$ values varying from $5-$ $320 \mathrm{MPa}$ (highly flexible) [80, 81] to $2-14 \mathrm{GPa}$ (very rigid) [82, 83] have been reported.

To clarify this issue, Fitzpatrick et al. [84] used 4D-EM to directly and noninvasively visualize the oscillatory dynamics of individual free standing VQIVYK amyloid nanocrystals (see Fig. 9a-left). When the proteins are bound with a suitable chromophore (dye Orange $\mathrm{G}$ in this case), the pump laser beam is sufficiently attenuated while passing through the nanocrystal and the material undergoes an inhomogeneous expansion as a result of photon absorption. This mechanism is able to initiate the oscillations of this "amyloid cantilever" (Fig. 9a-left). Considering that the frequency of a cantilever is proportional to the square-root of the Young modulus of the material, from the determination of the lowest eigenmodes frequency, coupled with a finite element analysis performed on the 3D tomographic reconstructions of the investigated crystals (see Fig. 9b-left), the authors were able to accurately determine the value of the Young modulus of the hydrogen-bonded cross- $\beta$ structure, $Y \approx 1 \mathrm{GPa}$. This exceptional rigidity of amyloid is thought to be responsible for the stiffening of normally healthy elastic tissues, thus disrupting normal cellular function. Both extracellular and intracellular distortions of flexible cell membranes by rigid amyloids (see schematics in Fig. 9a-right 
and 9b-right) may lead to leakage and ultimately cell death, possibly playing a crucial role in the pathogenesis of Alzheimer's disease [85].

Besides protein crystals, controlled organization of biological materials, such as DNA molecules, has attracted a great interest in recent years $[6,86]$ because of their natural tendency to assemble into complex nanosystems and nanomachines with active and specialized functions. The robustness and the mechanical motions of their primary components is thus fundamental for the stability and functionality of the assembled nanostructures. In this context, Lorenz and Zewail [87] used 4D-EM to investigate the mechanical properties of a freestanding DNA nanostructure by direct visualization of its vibrational oscillations (see Fig. 9c). Because DNA does not significantly absorb light, the oscillations were excited by an impulsive strain burst created by the laser pump in the carbon substrate, with a sufficiently broad frequency-spectrum such that the eigenmodes of the DNA nanostructure could be excited. From the measured oscillatory dynamics, the authors were able to distinguish between collective low-frequency modes $(\sim 4 \mathrm{MHz}$ and $\sim 8 \mathrm{MHz})$ and high-frequency vibrations of individual filaments $(\sim 15 \mathrm{MHz}$ and $\sim 16 \mathrm{MHz}$, Fig. 9c). Using the mathematical description for the case of a single cantilever ( $Y \propto f^{2}$, where $f$ is the eigenmode frequency), the Young modulus of DNA was obtained from the oscillations of the individual filaments. With $f=15-16 \mathrm{MHz}$ the authors derived $Y \approx 11-15 \mathrm{GPa}$, which is considerably higher than the value of $300 \mathrm{MPa}$ reported for DNA molecules in solution. This stiffening can be associated with a consistent dehydration of the investigated DNA filaments [88]. These results, together with the $4 \mathrm{D}$ cryo-EM observations of insulin fibrils described above, open 
up the possibility of controlling the mechanical properties of biomaterials by modulating their water content.

\section{4. $4 D$ structural dynamics of amyloid}

Another dimension to 4D-EM for the study of the mechanical properties of biological systems, besides the "bio-cantilever" experiments discussed above, comes from the ability to selectively probe unit cell deformations along different directions with sub-picometer resolution. An atomic scale view of the elastic behavior of biomaterials is, in fact, crucial to fully elucidate their function in living organisms. In a recent publication, Fitzpatrick et al. [21] exploited the exquisite sensitivity of $4 \mathrm{D}$ electron diffraction to directly probe the bond stiffness of the intermolecular forces stabilizing amyloid proteins at the atomic level.

The authors applied a laser-induced temperature jump to an individual VQIVYK amyloid submicrometer crystal (see Fig. 10a), properly bound to a suitable chromophore (dye Orange $G$ ) for efficient heat transfer. By monitoring the $\langle 110\rangle$ Bragg reflection (see Fig. 10b-left), they were able to measure the relative expansion of the unit cell along the stacking direction of the hydrogen-bonded $\beta$-strands present within a single $\beta$-sheet (intrasheet direction). From the measured strain, $\varepsilon$, and considering that the stiffness, $k$, is proportional to $\frac{1}{\varepsilon^{2}}$, an intrasheet bonding stiffness of $k_{\text {intrasheet }}=2.0 \mathrm{~N} / \mathrm{m}$ was obtained. This value is very high and similar to that of other cooperatively hydrogen-bonded materials $\left(k_{H-\text { bond }}=2-3 \mathrm{~N} / \mathrm{m}\right)$, such as ice [89]. This suggests that amyloid has an exceptional intrasheet rigidity, as also revealed by the oscillatory dynamics discussed in the previous section [84] where a Young modulus of $\sim 1 \mathrm{GPa}$ was determined, which arises mainly 
from generic interbackbone hydrogen bonding.

Besides the stacking of hydrogen-bonded $\beta$-strands, the hierarchical structure of amyloid is stabilized by two additional main protein-protein interfaces, each of them giving rise to characteristic Bragg reflections: $(i)$ a cross- $\beta$-sheet packing into a multisheet protofilament (intersheet), and (ii) the lateral association of protofilaments (interprotofilament). By tilting the single crystal shown in Fig. 10a with respect to the electron beam such that it can be probed along the [110] zone axis, the $(00 l)$ reflections associated to the interprotofilament direction become visible together with the (110) spots of the intrasheet direction. From the measured dynamics (see Fig. 11, lower panel), a 2.5-times larger relative expansion was obtained for the interprotofilament direction with respect to the intrasheet, thus resulting in a considerably smaller bonding stiffness, $k_{\text {interprotofilament }}=0.14 \mathrm{~N} / \mathrm{m}$. This is consistent with the combination of dipole-dipole electrostatic attraction and weak, bifurcated hydrogen bonds at the protofilament-protofilament interface.

To probe the sidechain-sidechain, intersheet direction the authors monitored the diffraction pattern of an ensemble of crystals, rather than that of a single crystal, and exploited the greater number of reflections available in this case to also resolve the (802) reflection at $0.52 \mathrm{~nm}$ associated to the intersheet direction. The observed dynamics (see Fig. 11, upper panel) resulted in a laser-induced expansion along the intersheet direction over 4 times larger than that along the intrasheet direction, meaning that the hydrogenbonding network within the $\beta$-sheets is almost 20 times more rigid than the cross- $\beta$-sheet packing. This gives a bonding stiffness $k_{\text {intersheet }}=0.07 \mathrm{~N} / \mathrm{m}$, which is consistent with an amphiphilic interaction between hydrophobic and 
hydrophilic sidechains.

This pronounced elastic anisotropy, where the stiffness of longitudinal hydrogen bonding (intrasheet), $k_{\text {long }}$, is almost 20 times larger than the stiffness of transverse amphiphilic and electrostatic interactions, $k_{\text {lat }}$, is likely to induce a length-dependent mechanical behavior of amyloid. This is particularly important in the bending of individual fibrils, which involves stretching of longitudinal bonds and shearing of transverse intersheet and interprotofilament bonds. By analogy with cytoskeletal bundle mechanics [90], a protofilament coupling parameter, $\gamma$, can be defined as a measure of the competition between intersheet/interprotofilament shearing and fibril stretching: $\gamma=\frac{k_{\text {lat }}}{k_{\text {long }}}\left(\frac{L^{2}}{\delta^{2}}\right)$, where $\frac{k_{\text {lat }}}{k_{\text {long }}}=0.05, L$ is the fibril length and $\delta$ is the axial spacing of intersheet/interprotofilament bonds $(\delta=4.8 \AA)$. Therefore, when $\gamma$ is smaller than $N$, the total number of intersheet and interprotofilament interfaces per layer of fibril (generally between 10 and 20), and thus the fibrils are below a critical length $L^{*} \sim 10 \mathrm{~nm}$, the deformation induced by the bending would be accommodated by both stretching and shearing. However, when $\gamma$ is larger than $N$ and thus $L^{*}$ larger than $10 \mathrm{~nm}$, only longitudinal stretching would take place. This results in very flexible and ductile short fibrils, whereas longer fibrils become stiff and brittle. Because the level of cytotoxicity depends on the degree of stiffness [7], these results are particularly relevant for the understanding of fibril-membrane interactions and fragmentation mechanisms, which play a crucial role in the spread and pathogenesis of amyloid diseases. 


\section{Conclusions and perspectives}

In this review, we have painted a portrait of four-dimensional (4D) electron microscopy and its applications in materials science and biology (see Fig. 12). Ultrafast imaging, e-diffraction and e-spectroscopy were all used to elucidate the dynamical behavior of complex organic, inorganic and biological structures in a multi- and inter-disciplinary approach. In our excursus, we were able to highlight different phenomena, from atomic motions during structural dynamics to transient near-field visualization, from phase transitions to carrier transport, and lastly from biological imaging to cryo-EM and biomechanics. The spatiotemporal investigations of matter presented here represent the foundation for our understanding of the molecular mechanisms behind complex biological processes, and in the rational design of complex systems.

Because of the versatility of 4D-EM for different materials, sample geometry and experimental conditions, its future applications are promised to be endless. On the materials science level, the strategic objective for

the near future would be a full coherent control of the different degrees of freedom of the structure involved, in order to manipulate their nonequilibrium properties from the atomic scale to the mesoscale. In recent years, in strongly-correlated systems, where a strong interaction between atomic and electronic degrees of freedom takes place, mode-selective control using $\mathrm{THz}$ light pulses has shown the potential to induce substantial changes of their electronic and magnetic properties with subtle modulation of the lattice structure [91]. Using resonant excitation of vibrational modes by $\mathrm{THz}$ fields, engineering of transient states of matter, not achievable at equilib- 
rium, could be reached. In multiferroics, superconductors, graphene and 2D monolayers of transition-metal-dichalcogenides, this can lead to novel electronic and magnetic properties that can only be probed when high spatial, temporal and energy resolutions are invoked, as shown here using 4D-EM.

From a biological perspective, the next step would be the possibility to investigate the dynamical processes of complex biomaterials and molecular structures in living cells, using, for instance, a liquid cell or the cryo-electron microscopy configuration. This would provide crucial information on how their properties may relate to the pathology in native conditions and the mechanisms regulating their function. The four-dimensional approach presented here would make possible the recording of snapshots of the multitude of conformational intermediates in action, making movies of real-time molecular processes with both high spatial and temporal resolutions [92]. For recent collected works from this group on current state-of-the-art in this field, the reader is referred to the book by Zewail [11] entitled " $4 \mathrm{D}$ Visualization of Matter".

\section{Acknowledgements}

This work was supported by the National Science Foundation and the Air Force Office of Scientific Research in the Center for Physical Biology at Caltech supported by the Gordon and Betty Moore Foundation. 


\section{Figure Captions}

Figure 1. Four-dimensional (4D) electron microscopy. (a) Schematic representation of the integration of the laser systems to a redesigned transmission electron microscope, equipped with two ports for optical access. A pulsed

laser delivers a train of optical pulses (fs/ps/ns); a portion of the laser output is guided to the photocathode of the electron microscope and generates electron packets which are used to probe the investigated sample. The dynamics of interest are initiated by the second output portion of the laser, which is delayed with respect to the electron probe using an optical delay stage. The electron beam after interaction with the sample is then recorded on a detection system allowing imaging, diffraction and spectroscopy. Adapted from Lobastov et al. [12] (Copyright 2005 National Academy of Sciences, USA). (b) Experimental layout for single nanoparticles investigation in the 4D microscope equipped with electron energy analyzer. By inserting a small aperture in the image plane, single-particle selectivity can be achieved and imaging, diffraction and spectroscopy can be performed. Adapted from van der Veen et al. [17], and from van der Veen et al. [35] (used in accordance with the Creative Common Attribution 3.0 Unported License).

Figure 2. Ballistic and diffusive phonon transport in GaAs quantum dots. (a) Schematic layout of the photon-pump/electron-probe 4D diffraction experiment in the reflection geometry. (b) Experimental (full symbols) and simulated (solid lines) dependence on the dot size and excitation fluence of the time constant, $\tau_{d}$, associated with phonon-phonon interactions; $\tau_{\text {anh }}=3.5 \mathrm{ps}$ is the time constant of anharmonic decay of optical-phonons toward high- 
frequency (hot) acoustic phonons; $\Lambda_{p}=26-27 \mathrm{~nm}$ is the phonon inelastic mean-free path in GaAs. (c-d, left) Space-resolved maps of the time constant $\tau_{d}$, as extracted from experiments and calculations, for dot size smaller (c) and larger (d) than $\Lambda_{p}$. For dot size smaller than $\Lambda_{p}$ (height, $h=11 \mathrm{~nm}$ ), the absence of additional decay components, besides the optical-phonon decay in $\tau_{a n h}$, indicates that hot acoustic phonons travel unperturbed within the dots (no collisions, ballistic transport). For dot size larger than $\Lambda_{p}$ (height, $h=46 \mathrm{~nm}$ ), phonon-phonon scattering in the acoustic population, which lengthen the time constant $\tau_{d}$, dominates (diffusive regime). (c-d, right) Transient behavior of the interatomic distance measured for dot size smaller (c) and larger (d) than $\Lambda_{p}$. For 11-nm dots, the observation of coherent oscillations confirms that phase correlation is preserved during ballistic phonon propagation (coherent transport). Adapted with permission from Vanacore et al. [26] (Copyright 2014 American Chemical Society).

Figure 3. Imaging of localized near-fields. (a) Schematic of Photon-induced near-field electron microscopy (PINEM). A fs optical pulse (with electric field depicted by the green sine wave) interacts with a nanoparticle, whose size is much smaller than the optical wavelength. This generates a local electric field, schematically represented in the xz-plane, as a result of surface evanescent excitation. An ultrashort electron pulse, synchronized with the photon pulse, is inelastically scattered by the component of the near-field along the electron's propagation direction (z-axis), inducing a quantized energy exchange and satisfying the uncertainty principle for the coupling involving the electron, the photon and the nanostructure. (b) Experimental PINEM 
image of a $100 \mathrm{~nm}$ silver nanoparticle on a graphite substrate. The dipoleshaped field and the nodal plane (vanishing electric field) are evident. (c) and (d) PINEM images of two nanoparticles for two orthogonal light polarizations as indicated by the arrows. (e) Near-field of a nanoparticle pair with an edge-to-edge distance of $32 \mathrm{~nm}$. In this case, where their separation is smaller than the decay length of the evanescent fields, a void-field channel connecting the two particles is formed between them. (f) Near-field distribution observed inside and outside a triangular silver nanoparticle by selecting the first energy-gain peak $(+1 h \nu)$ when complete synchronization between optical and electron pulses occurs $(t=0)$. Here, a tightly focused electron beam, rather than parallel illumination like in panels (b)-(e), is used and scanned across the particle. Panels a and b are reproduced from Baskin and Zewail [48] (Copyright 2014 Elsevier Masson SAS. All rights reserved). Panels $\mathrm{c}$ and $\mathrm{d}$ are adapted with permission from Yurtsever and Zewail [44] (Copyright 2012 American Chemical Society). Panel e is adapted with permission from Yurtsever et al. [49] (Copyright 2012 American Chemical Society). Panel $\mathrm{f}$ is adapted from Yurtsever et al. [31] (Reprinted with permission from AAAS).

Figure 4. Visualization of nanofriction in space and time. (a, left) Experimentally measured behavior of the edge displacement for a $\mathrm{Cu}(\mathrm{TCNQ})$ crystal on an amorphous membrane (substrate). The subtraction of the intrinsic response of the crystal, due to intermolecular forces, from the observed dynamics allows one to isolate a periodic slip-stick behavior. The inset shows the sawtooth dynamics observed for the friction force. (a, right) Schematics 
of the relationship between the intrinsic restoring force and the slip-stick friction forces at the interface between the crystal and the substrate. Adapted with permission from Flannigan et al. [52] (Copyright 2010 American Chemical Society). (b) Single-shot 4D EM images of a $\mathrm{ZnO}$ nanotube partially filled with a lead core; scale bar, $100 \mathrm{~nm}$. When heated with a single laser pulse, the lead column expands before returning to its original length at long times. (c-d) Experimentally measured (c) and simulated (d) transient behavior of the lead column displacement within the $\mathrm{ZnO}$ tube. The friction coefficient is obtained by comparing the initial expansion with the results of an analytical model (blue line and dots in (c)). Adapted from Lorenz and Zewail [54] (Reprinted with permission from AAAS).

Figure 5. Single particle phase transition. (a) Bright-field images of a single $\mathrm{Fe}$ (pyrazine) $\mathrm{Pt}(\mathrm{CN})_{4}$ metal-organic framework nanocrystal, taken at different delay times after laser heating, undergoing a cooperative transition from a low-spin (LS) to a high-spin (HS) state. (b) Real-space anisotropic expansion dynamics of the single particle shown in (a) along two orthogonal directions. The blue and red plots correspond to the directions 1 and 2 in (a), respectively. (c) Reciprocal-space diffraction dynamics of the single particle shown in (a) under the same excitation conditions, the real-space experiment. Adapted from van der Veen et al. [17].

Figure 6. Spatiotemporal visualization of carrier transport in a p-n junction. (a) Schematic of scanning ultrafast EM experiment: optical pump initiates the carrier excitation, and transport of charges is imaged with a 
scanning electron probe at different delay times. (b) Experimentally measured temporal behavior of charge carriers at the surface of the diode. In the images, bright and dark contrasts correspond to excess in local electron and hole densities, respectively. The different frames shows: excitation $(+6.7$ ps), carrier separation after transport $(+36.7 \mathrm{ps},+80 \mathrm{ps})$, and relaxation to equilibrium (+653.3 ps, $3.32 \mathrm{~ns})$. The inset in the panel (a) shows the calculated charge density at the p-n junction interface. Adapted from Najafi et al. [62] (Reprinted with permission from AAAS).

Figure 7. PINEM imaging of protein vesicles and biological cells. (a) Brightfield TEM (left) and PINEM (right) images of protein vesicles. The PINEM image was recorded at the maximum overlap between the fs laser pulse and electron packet. (b) Polarization dependence of the spatial location of the contrast enhancement in PINEM images of protein vesicles. The polarization of the laser light is represented by the double-headed arrows. (c) Brightfield TEM (left) and PINEM (right) images of an unfixed and unstained Escherichia coli cell (scale bars, $500 \mathrm{~nm}$ ). Adapted from Flannigan et al. [71].

Figure 8. 4D cryo-electron microscopy of amyloid proteins. (a) Schematic representation of the four-dimensional cryo-electron microscopy experiment of biological specimens. (b) Real-space image (upper panel, scale bar of $2 \mu \mathrm{m}$ ) and selected area diffraction pattern (lower panel) of a network of insulin amyloid fibrils embedded in vitreous ice at $118 \mathrm{~K}$ on a lacey carbon substrate. In addition to the characteristic ring of the amyloids with equi- 
librium real-space separation of $0.48 \mathrm{~nm}$, the reflections of the vitreous ice with separations of $0.37 \mathrm{~nm}$ and $0.21 \mathrm{~nm}$ are also present. (c, left) Relative expansion of the hydrogen-bonded $\beta$-strands measured as a function of the delay time between the laser pump and the electron probe. (c, right) Schematics of the expansion of the fibrils embedded in glassy ice. The individual $\beta$ strands, connected by hydrogen bonds (black dashed lines), are shown as cyan ribbons. Adapted with permission from Fitzpatrick et al. [77] (Copyright 2013 American Chemical Society).

Figure 9. Biomechanics of amyloid proteins and DNA nanostructures. (a, left) Oscillatory dynamics of individual free standing amyloid nanocrystals. (b, left) Fourier transform of the periodic oscillations shown in (a) giving the frequency of the lowest eigenmodes. The directions of the major and minor oscillations are shown in the inset, together with the results of finite element analysis performed on the 3D tomographic reconstructions of the investigated nanocrystals. (a-b, right) Schematic representation of the extracellular and intracellular distortions of flexible cell membranes by rigid amyloids. (c) The left panel shows the vibrational oscillations of the free standing DNA nanostructure shown in the right panel. The displacement can be well described by a simple sinusoidal function. Panels a and b are adapted from Fitzpatrick et al. [84]. Panel $\mathrm{c}$ is adapted from Lorenz and Zewail [87].

Figure 10. Structural dynamics of a single amyloid crystal. (a) Real space image (left) and selected area diffraction pattern (center) of a single amyloid microcrystal. The structural dynamics is monitored by measuring a series of 
time-resolved patterns as a function of delay time between the laser pump and the electron probe (right panel). (b, left) Relative expansion of the hydrogenbonded $\beta$-strands within a single $\beta$-sheet (intrasheet direction) measured as a function of the delay time. (b, right) Schematic of the expansion along the intrasheet direction. The individual $\beta$ strands, connected by hydrogen bonds (black dashed lines), are shown as cyan ribbons. Adapted from Fitzpatrick et al. [21].

Figure 11. Mapping of amyloid intermolecular forces. (a) Anisotropic dynamics along the sidechain-sidechain, intersheet direction ((802) Bragg reflection) measured in an ensemble of crystals (upper panel), and along the interprotofilament direction ((004) Bragg reflection) measured in a single crystal (lower panel). In both cases the expansion along the intrasheet direction is monitored by probing the (110) reflection. (b) Schematics of the expansion along the intersheet (upper panel) and interprotofilament (lower panel) directions. The individual $\beta$-strands are shown as cyan ribbons, and the hydrogen bonds as black dashed lines. Adapted from Fitzpatrick et al. [21].

Figure 12. Pictorial illustration of the basic concept of four-dimensional (4D) electron microscopy, where time-resolved imaging, e-diffraction and espectroscopy represent its basic domains for probing matter. 


\section{D electron microscopy}
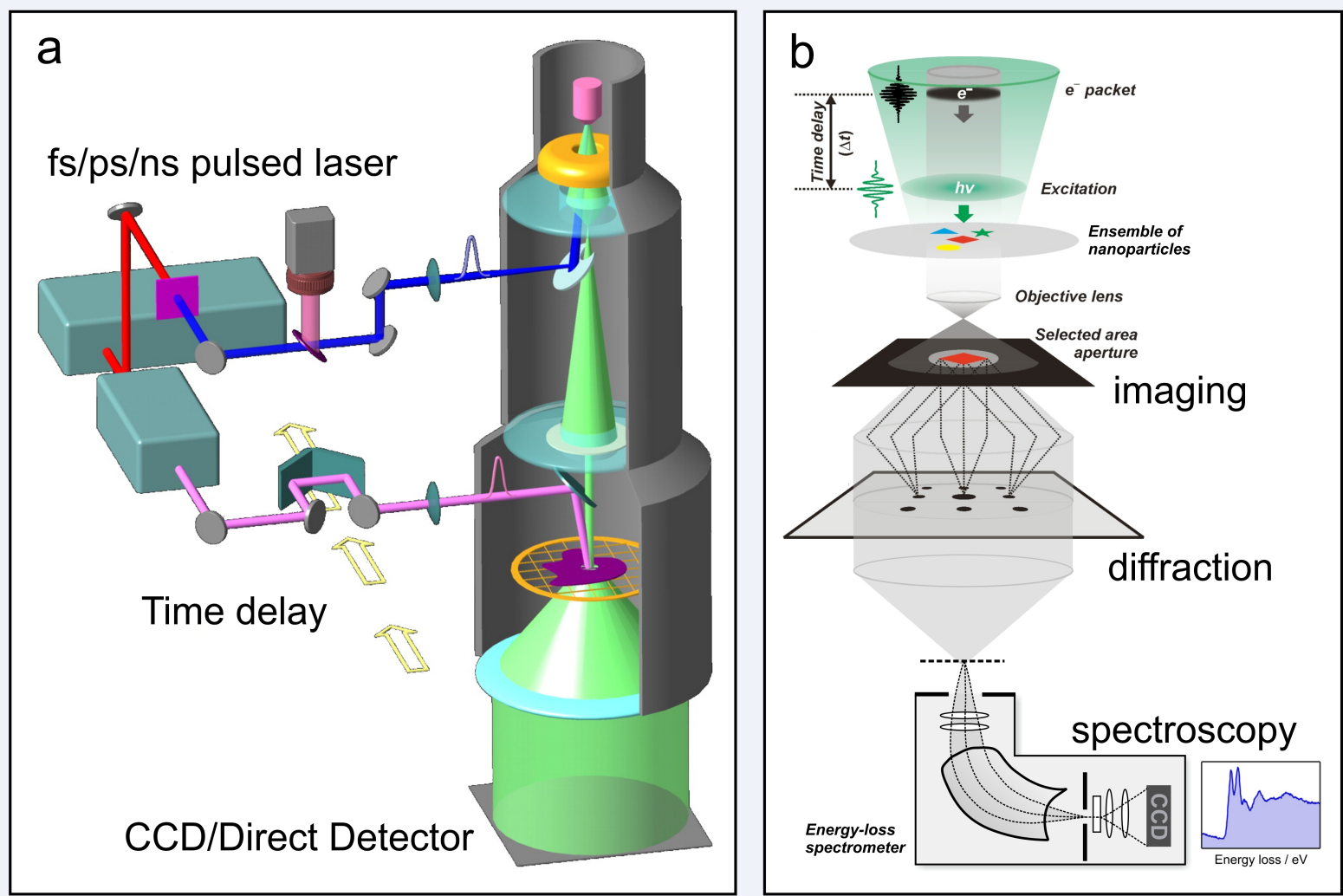

Figure 1: Vanacore, Fitzpatrick, and Zewail 
Phonon transport in quantum dots
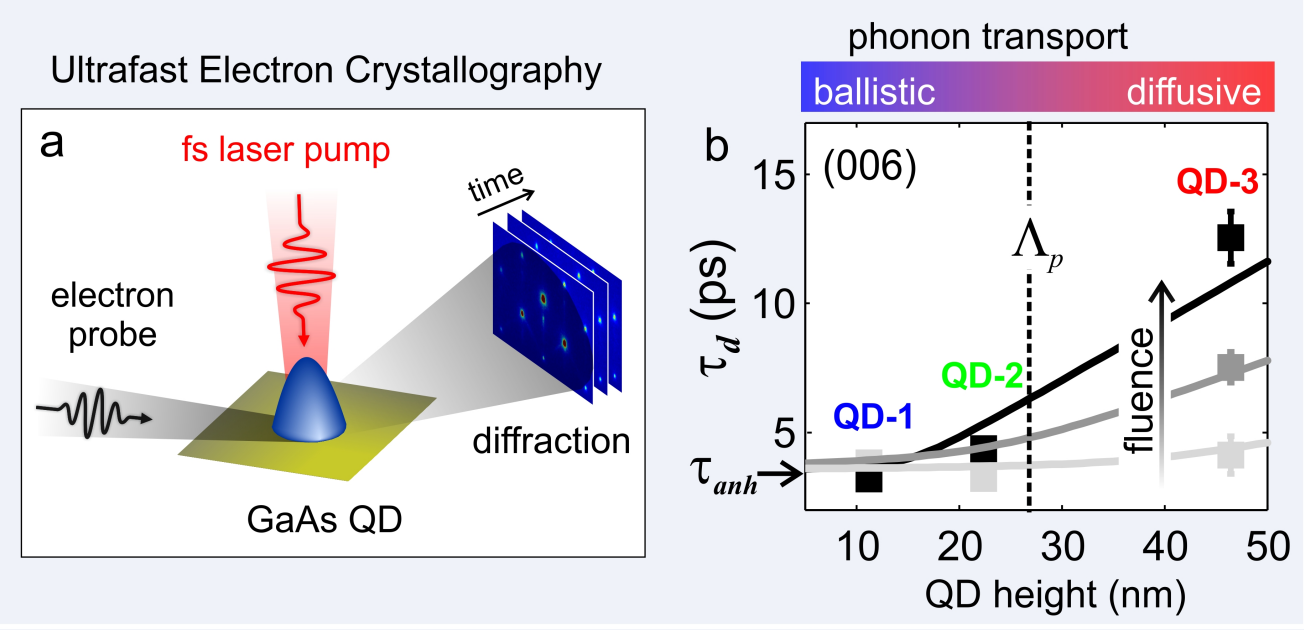

C

ballistic regime
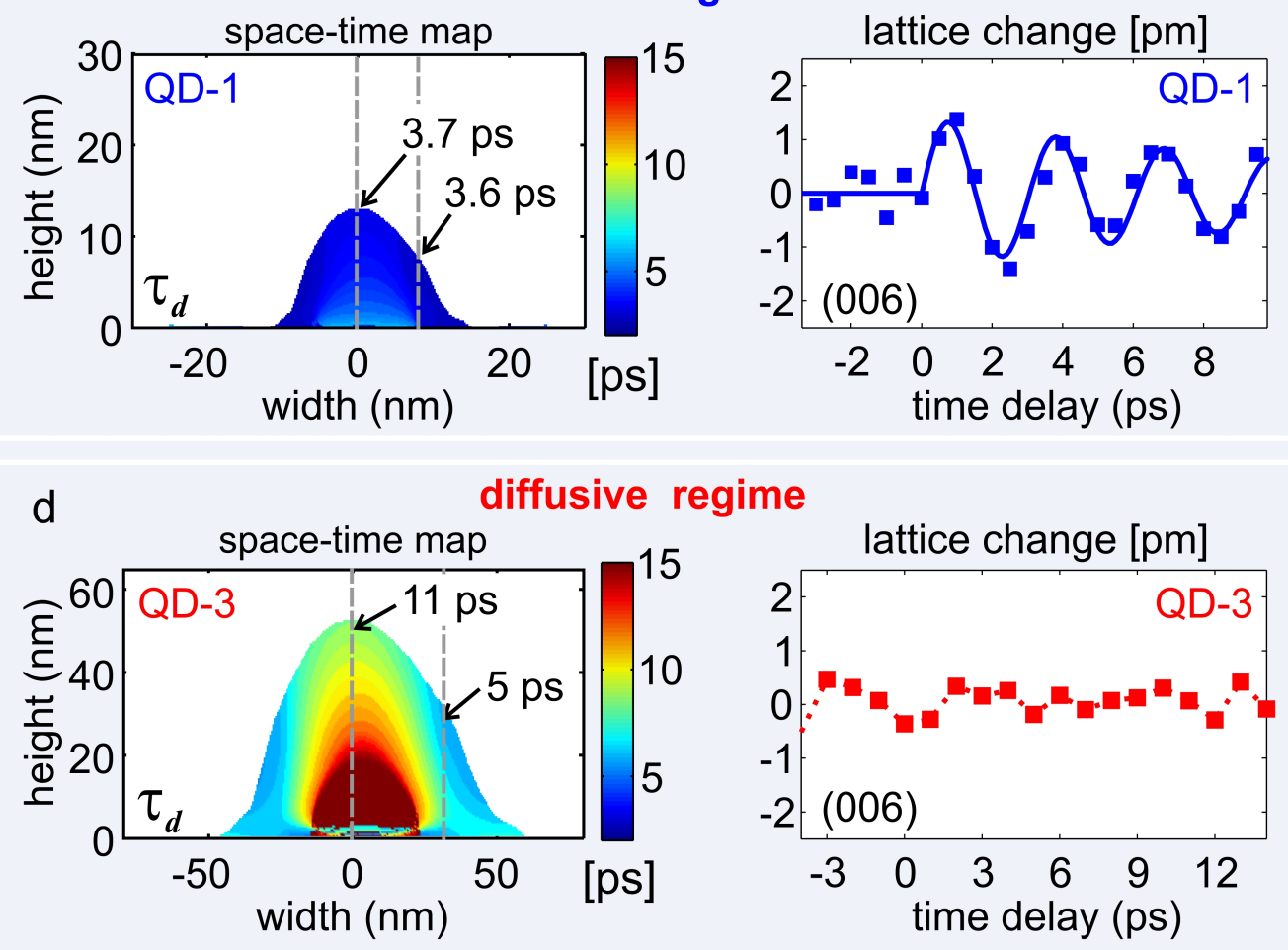

Figure 2: Vanacore, Fitzpatrick, and Zewail 


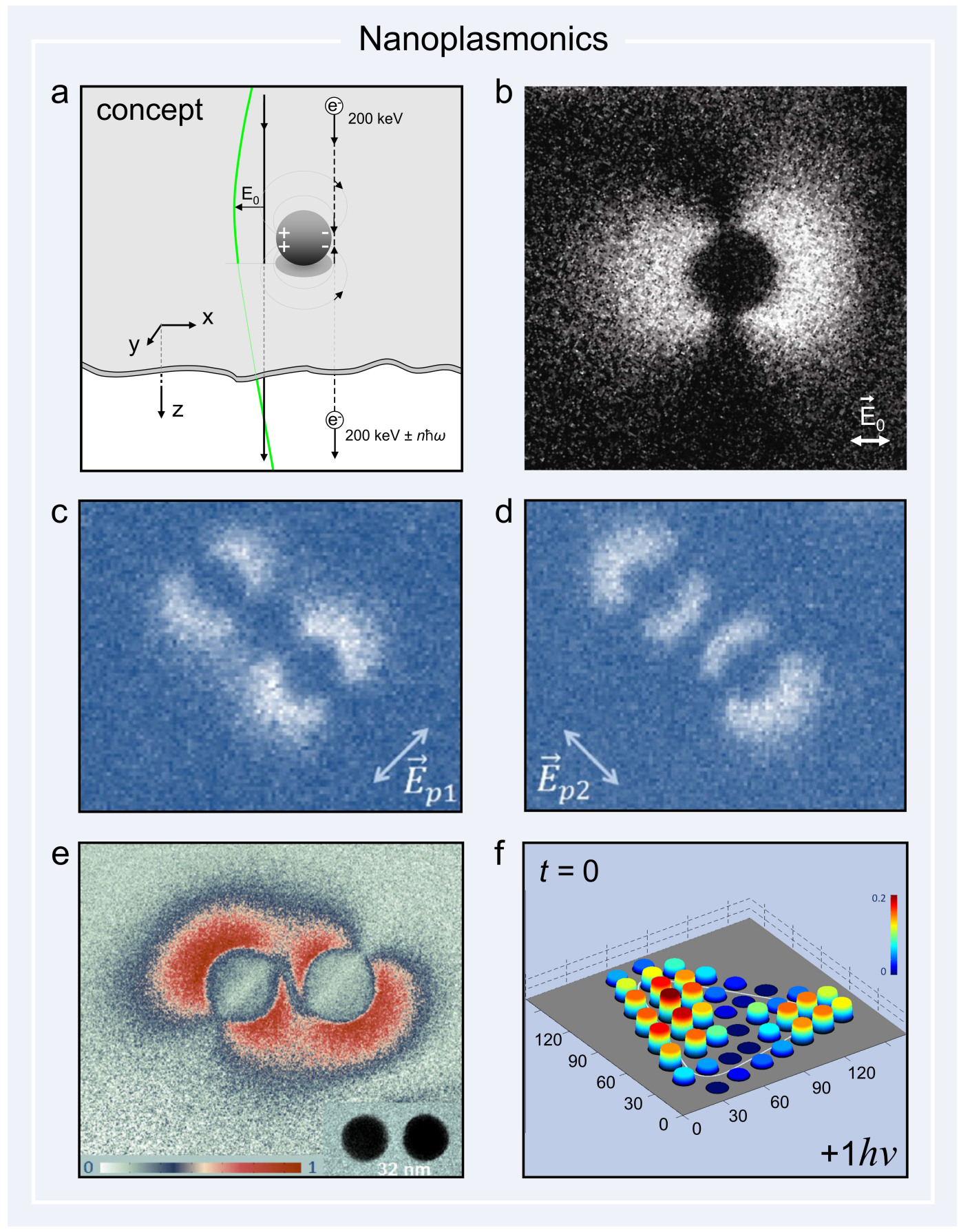

Figure 3: Vanacore, Fitzpatrick, and Zewail 


\section{Nanofriction}

a
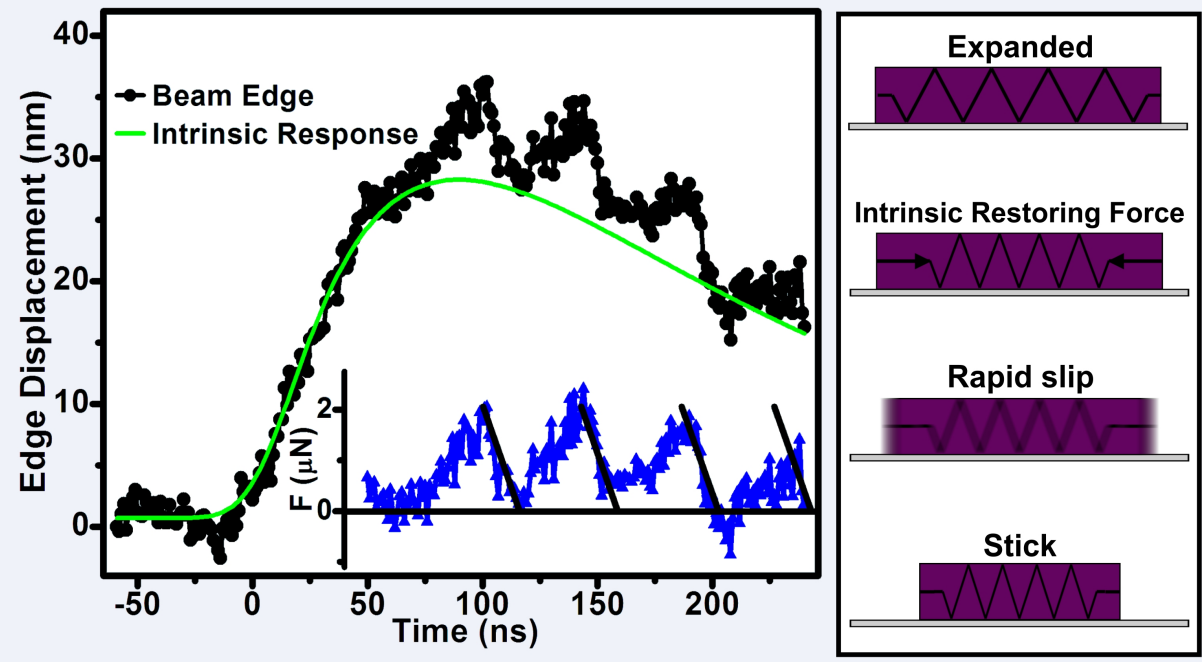

b
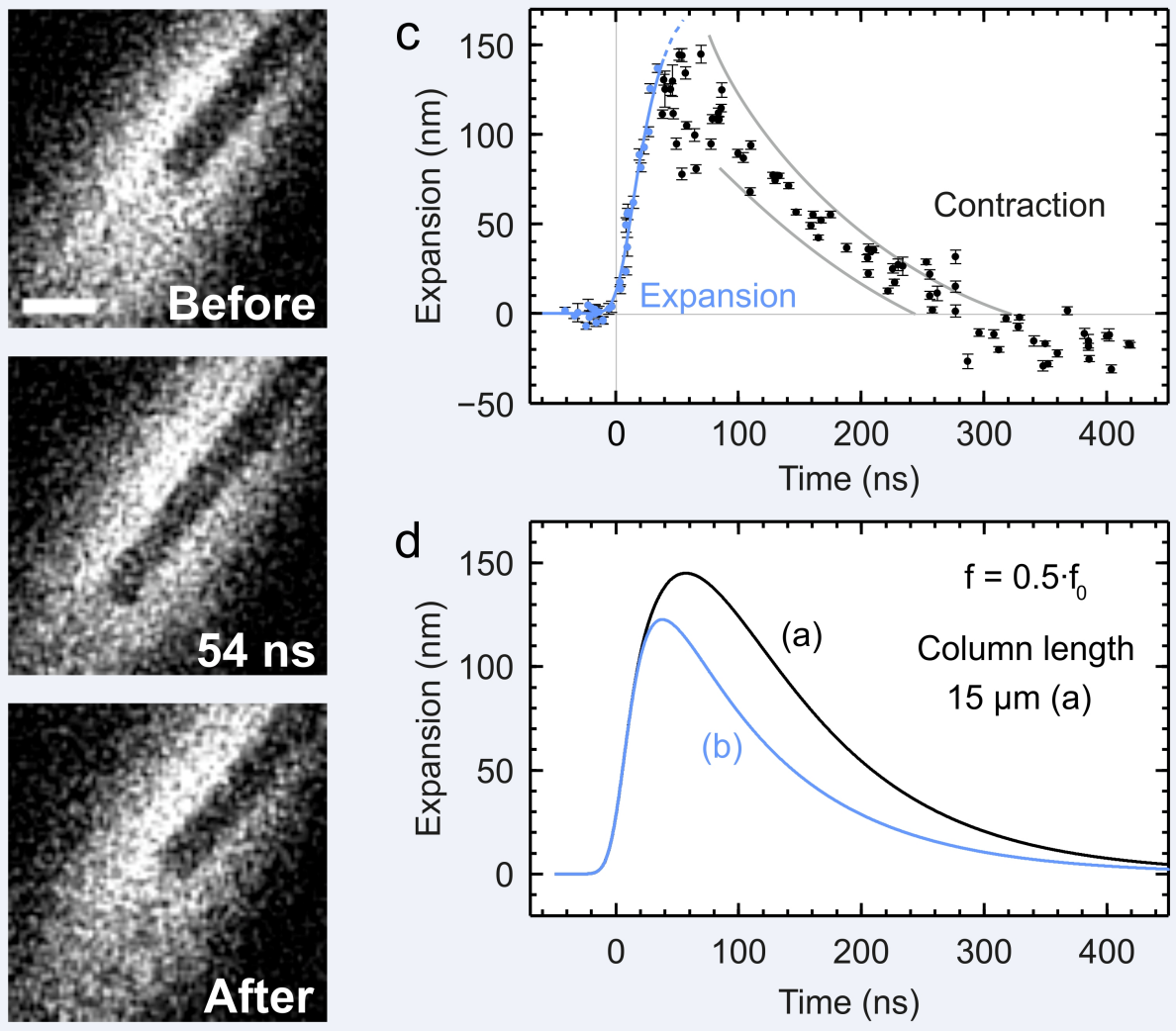

Figure 4: Vanacore, Fitzpatrick, and Zewail 
Single particle phase transition
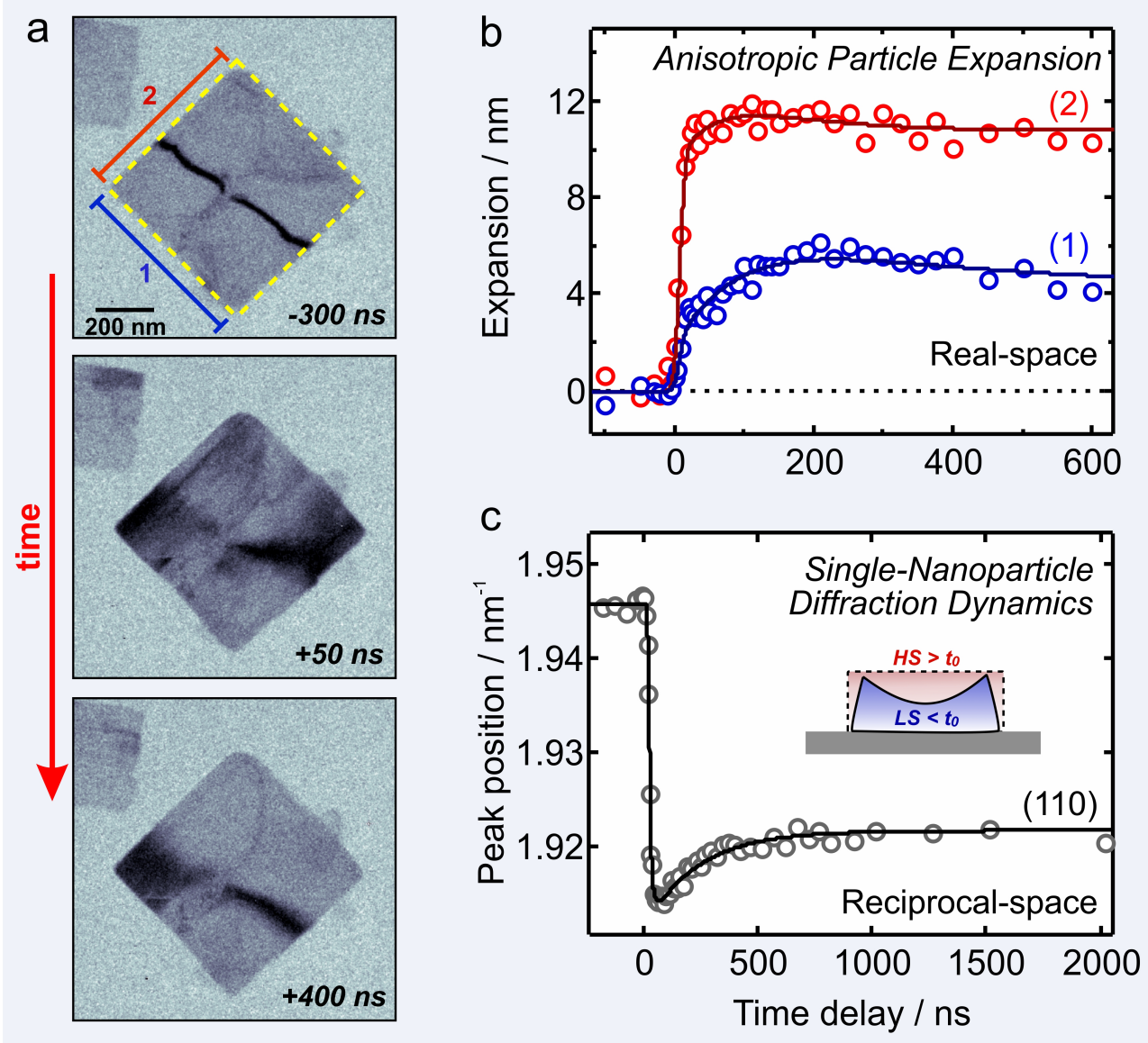

Figure 5: Vanacore, Fitzpatrick, and Zewail 


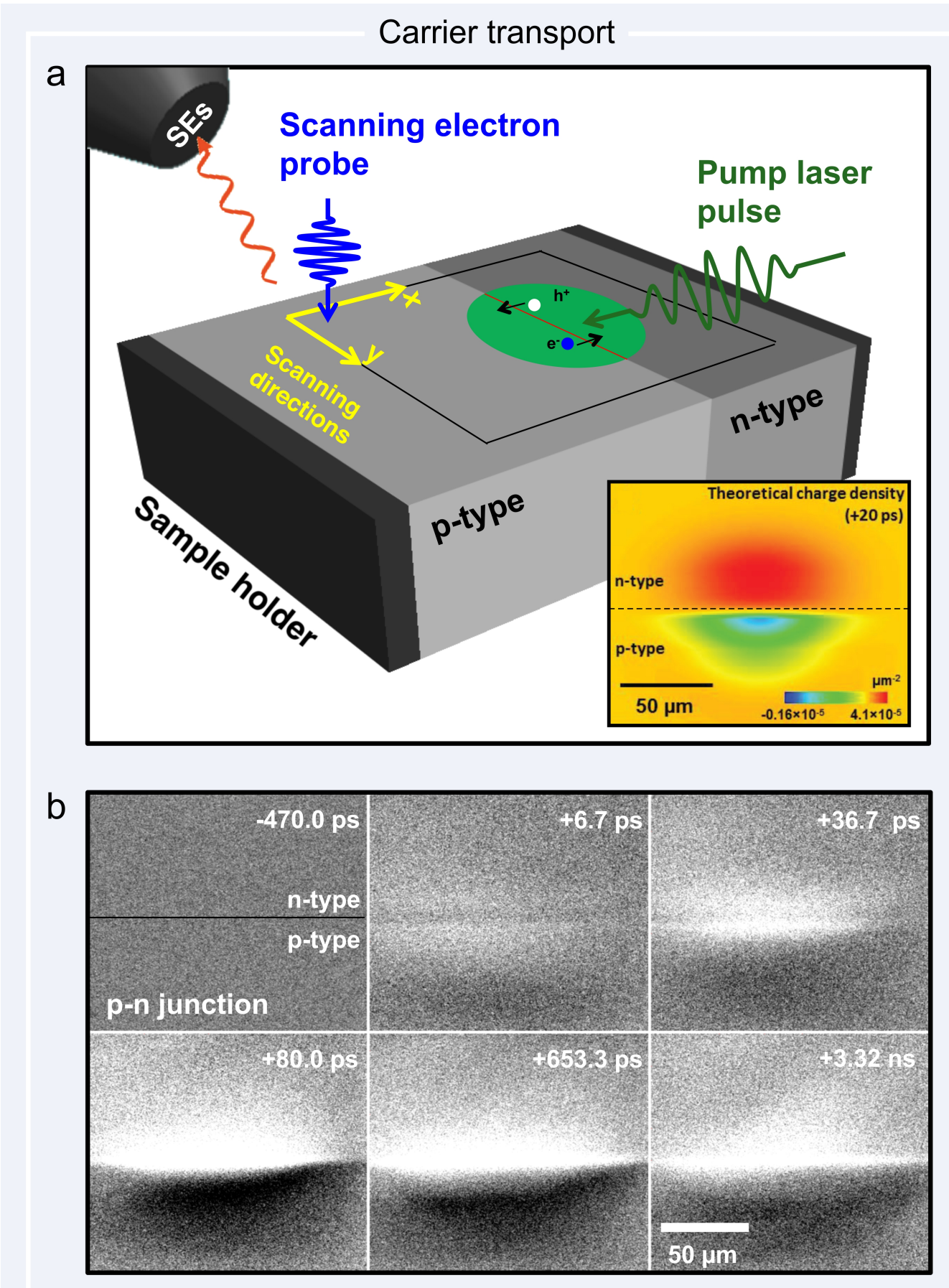

Figure 6: Vanacore, Fitzpatrick, and Zewail 
PINEM of protein vesicles and biological cells

a

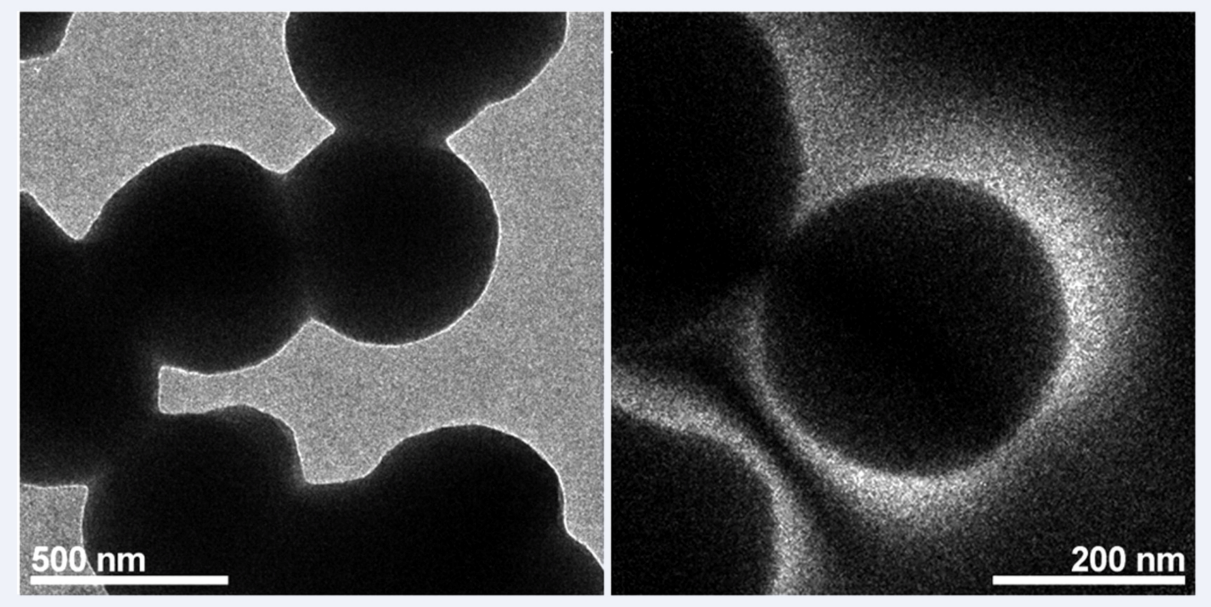

b
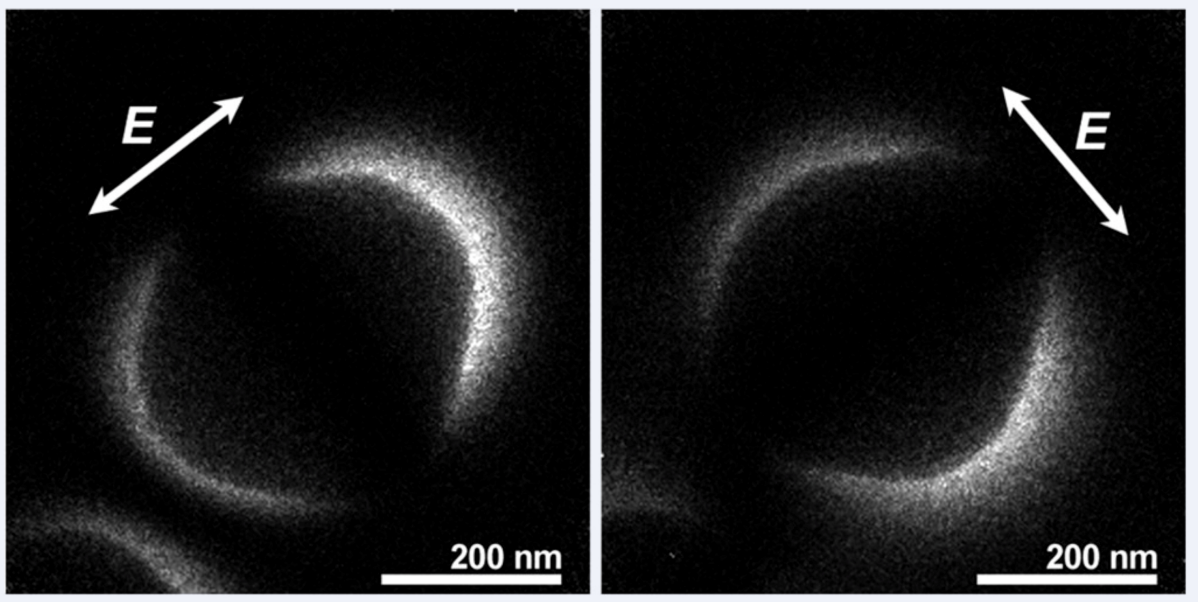

C

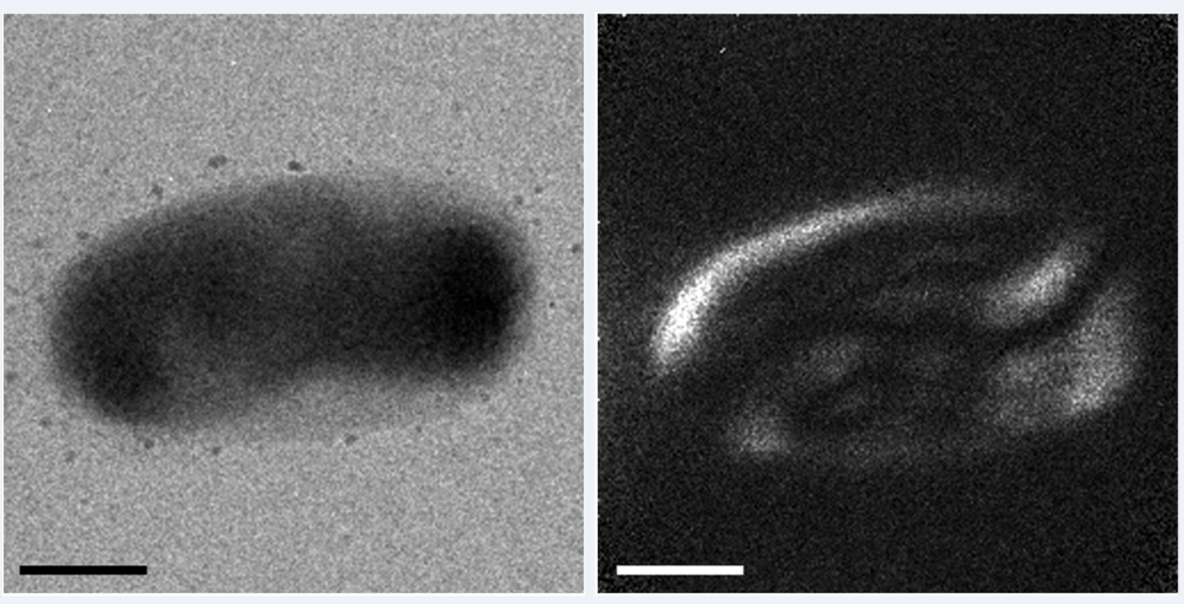

Figure 7: Vanacore, Fitzpatrick, and Zewail 


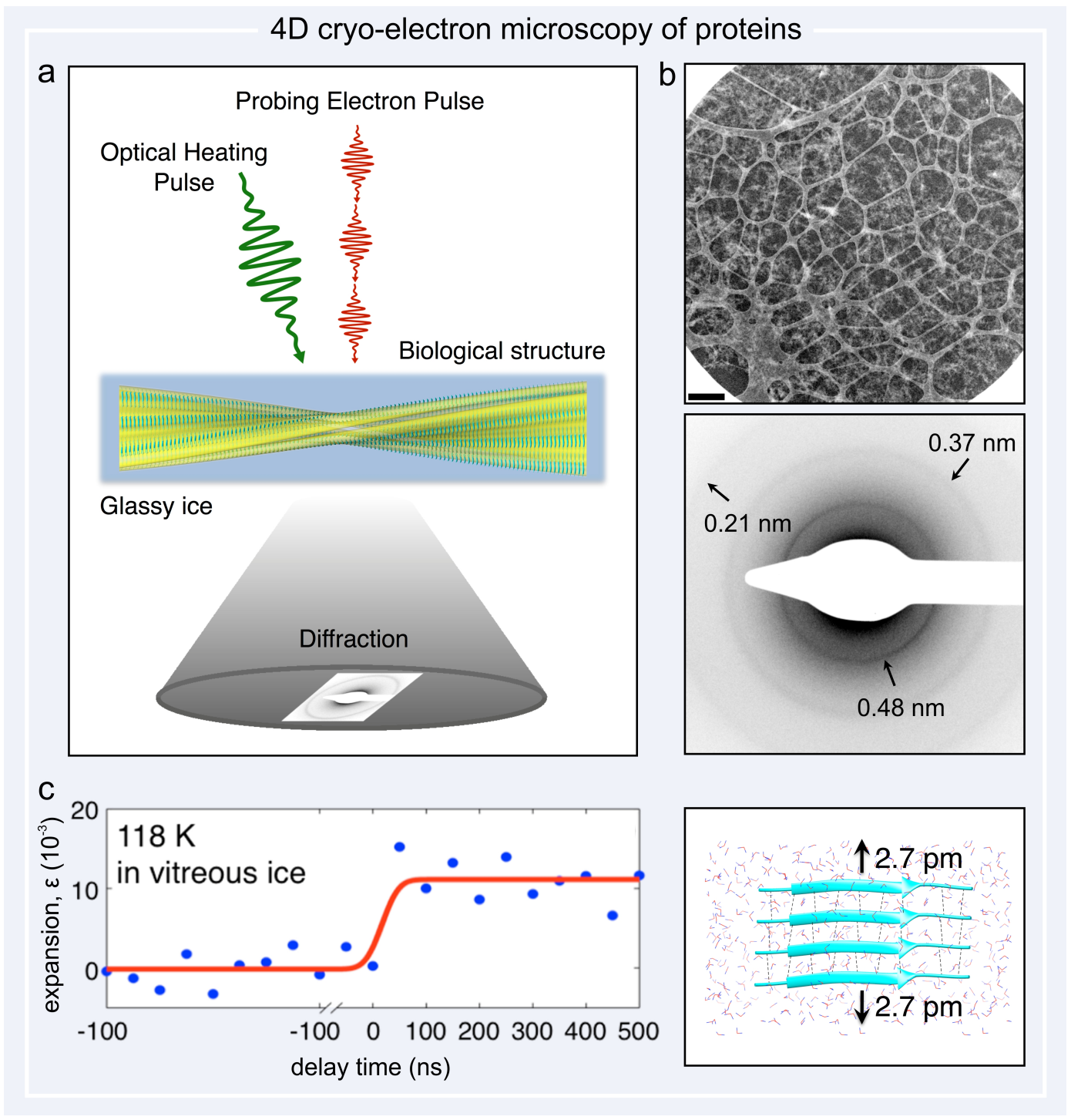

Figure 8: Vanacore, Fitzpatrick, and Zewail 


\section{Biomechanics}

a

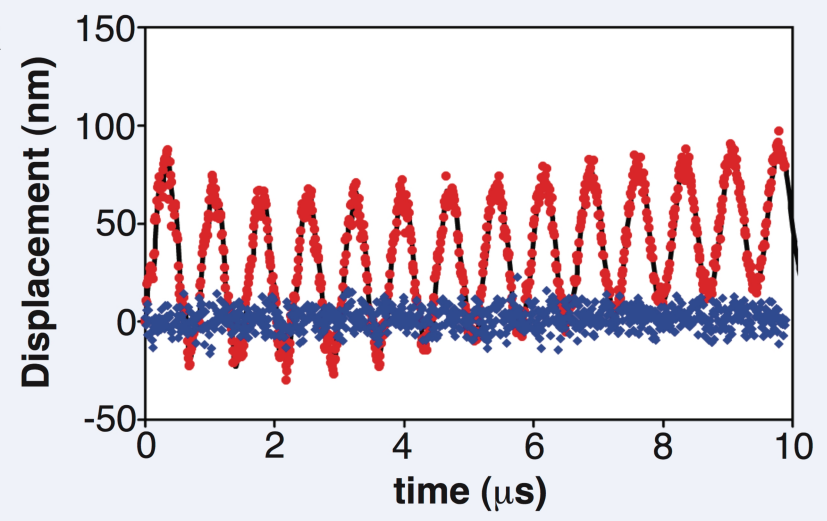

b
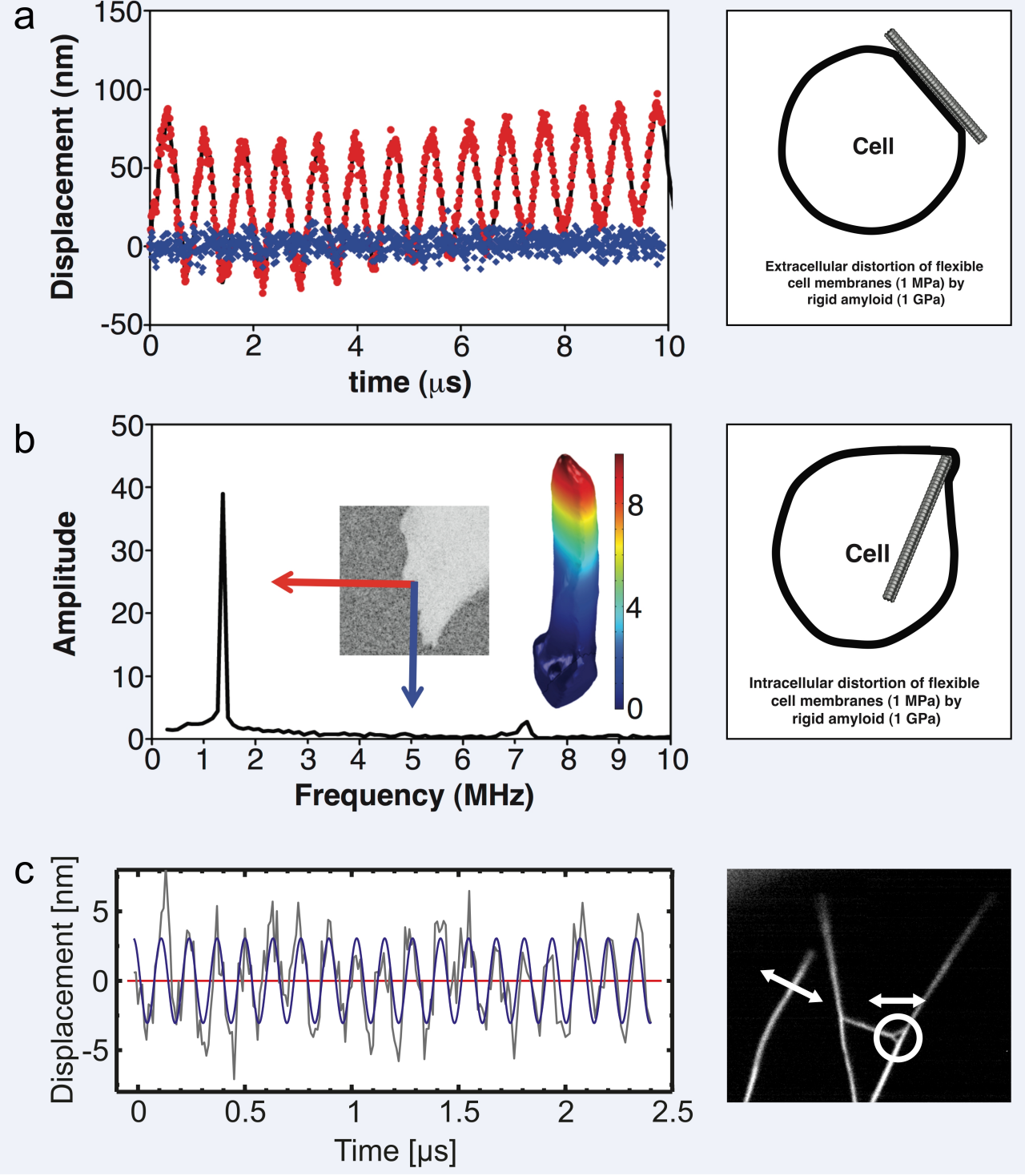

Figure 9: Vanacore, Fitzpatrick, and Zewail 


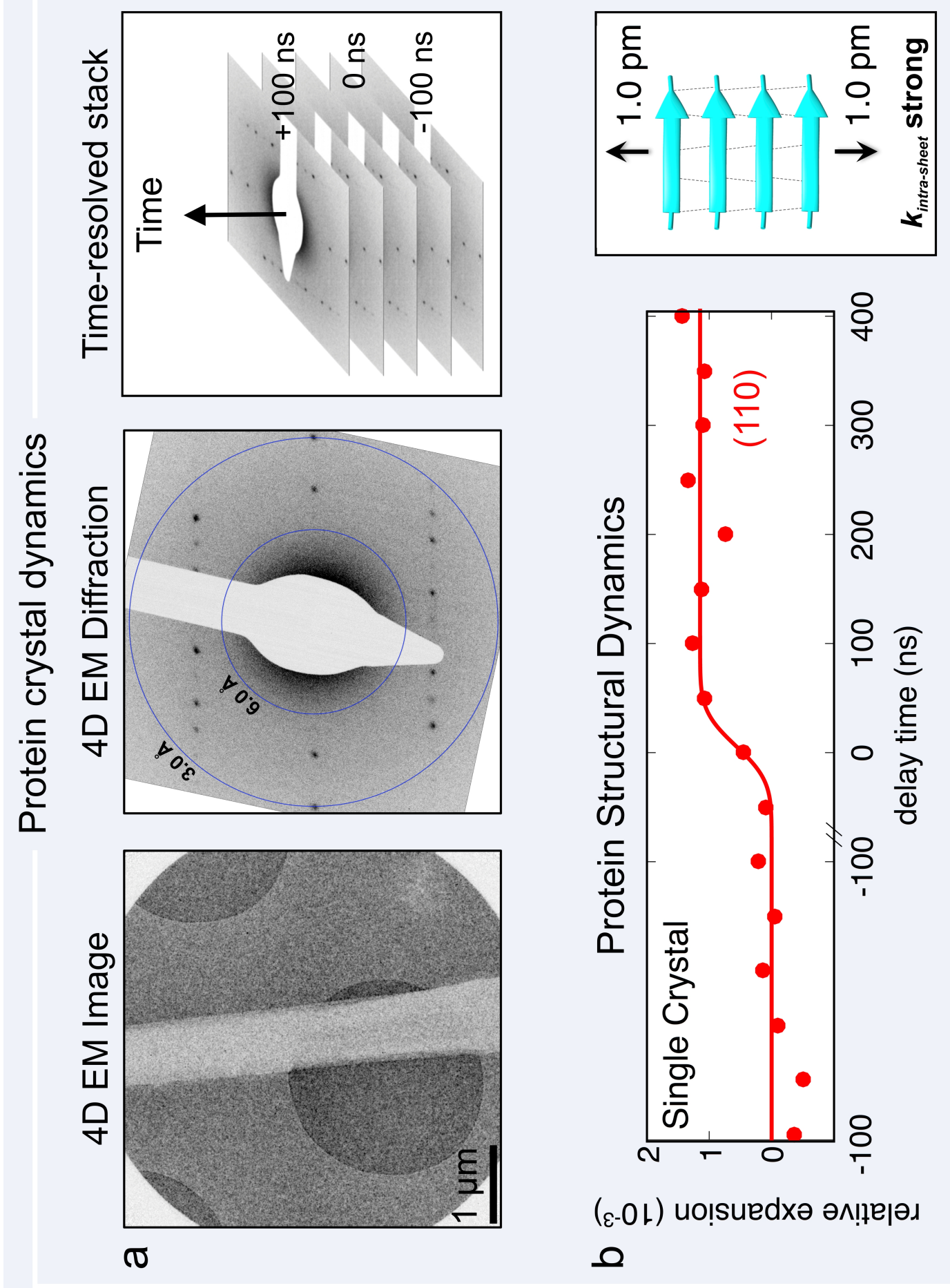




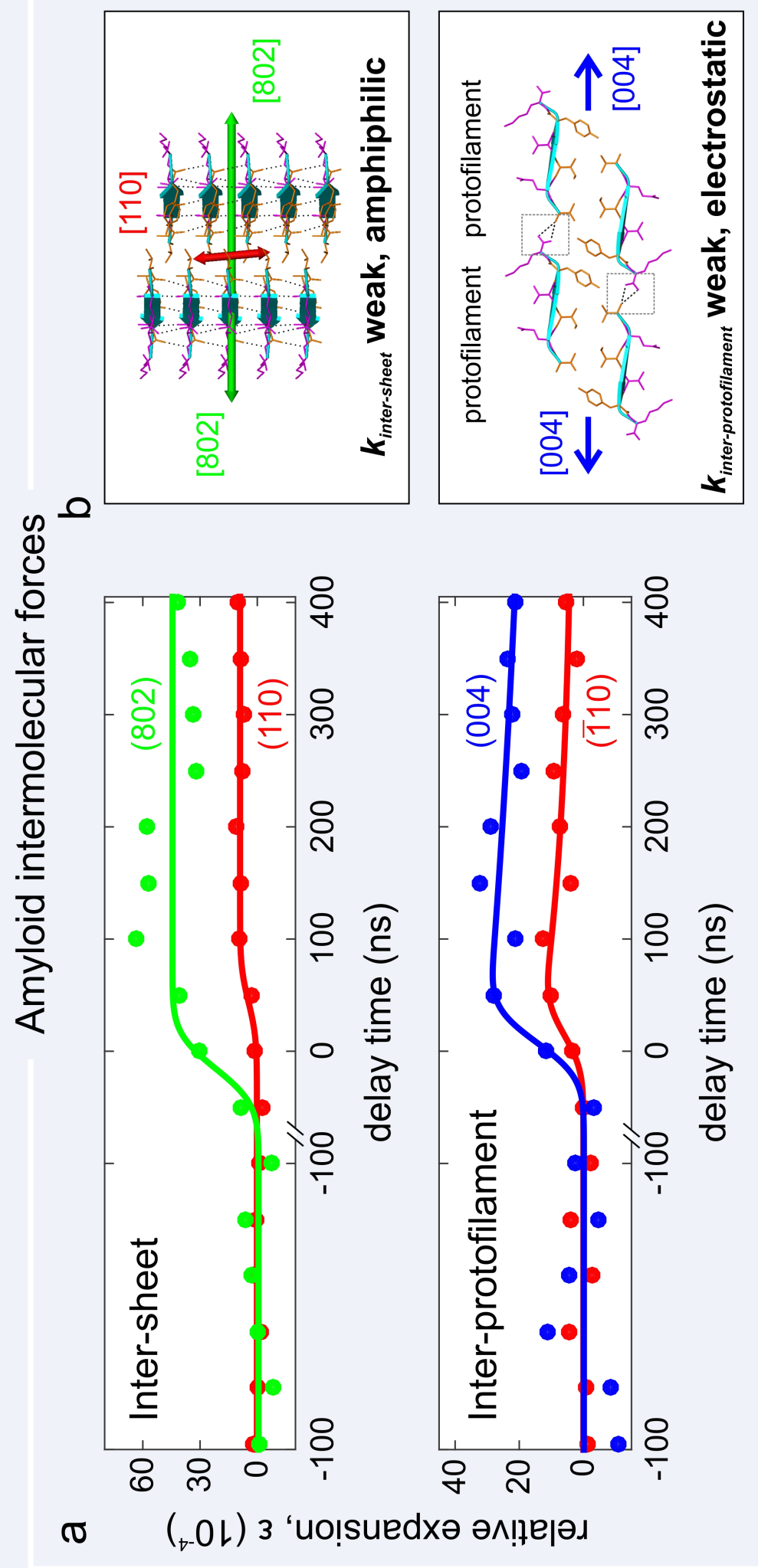

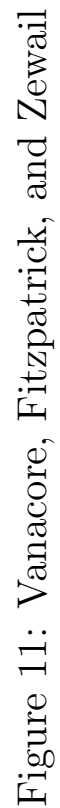




\section{Four-dimensional (4D) electron microscopy}

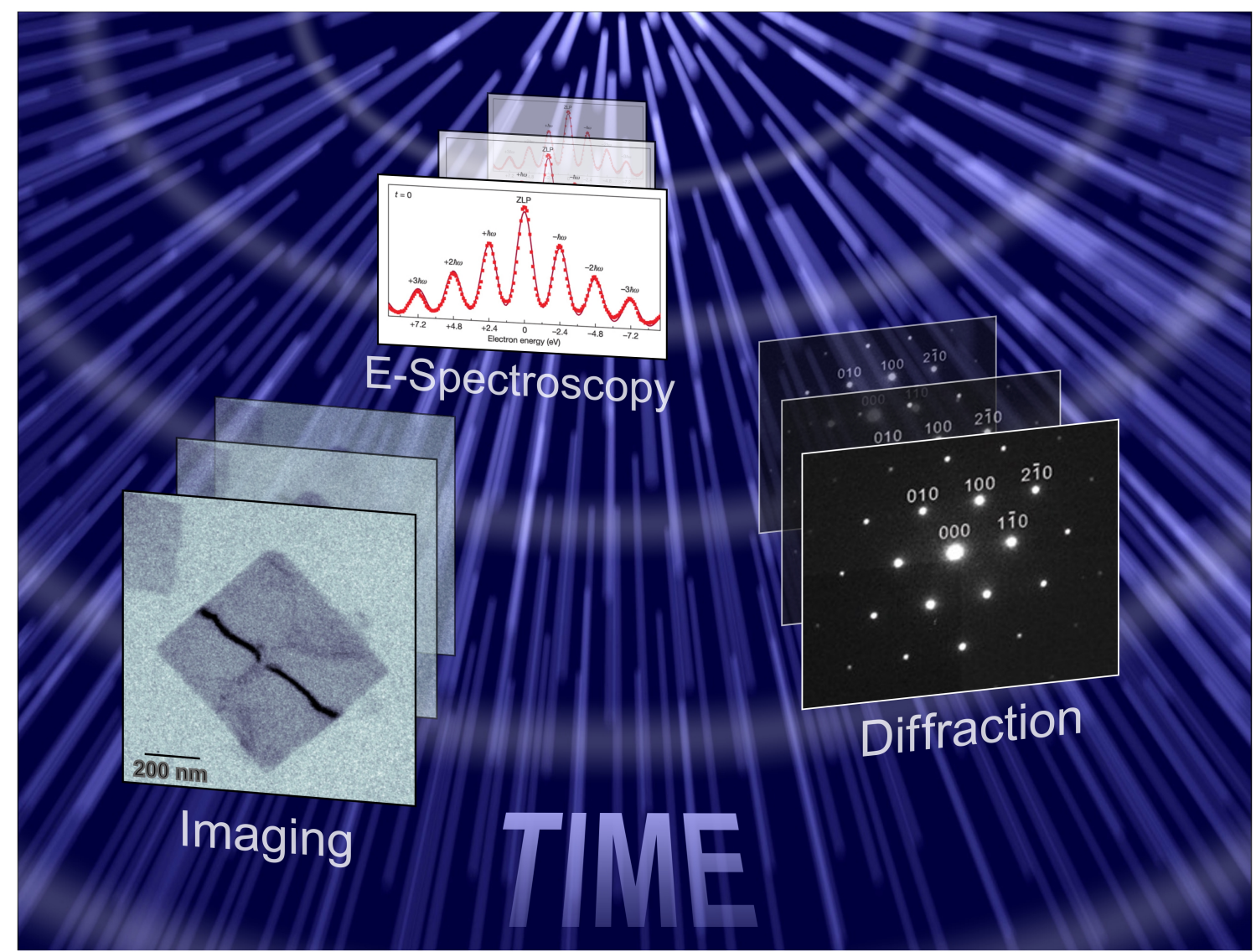

Figure 12: Vanacore, Fitzpatrick, and Zewail 


\section{Bibliography}

[1] M. Heiss, Y. Fontana, A. Gustafsson, G. Wst, C. Magen, D. D. O’Regan, J. W. Luo, B. Ketterer, S. Conesa-Boj, A. V. Kuhlmann, J. Houel, E. Russo-Averchi, J. R. Morante, M. Cantoni, N. Marzari, J. Arbiol,

A. Zunger, R. J. Warburton, A. Fontcuberta i Morral, Nat. Mater. 12 (2013) 439444.

[2] M. Maldovan, Nature 503 (2013) 209217.

[3] D. G. Cahill, P. V. Braun, G. Chen, D. R. Clarke, S. Fan, K. E. Goodson, P. Keblinski, W. P. King, G. D. Mahan, A. Majumdar, H. J. Maris, S. R. Phillpot, E. Pop, L. Shi, Appl. Phys. Rev. 1 (2014) 011305.

[4] F. Meinardi, A. Colombo, K. A. Velizhanin, R. Simonutti, M. Lorenzon, L. Beverina, R. Viswanatha, V. I. Klimov, S. Brovelli, Nat. Photon. 8 (2014) 392-399.

[5] P. Gao, J. Britson, J. R. Jokisaari, C. T. Nelson, S.-H. Baek, Y. Wang, C.-B. Eom, L.-Q. Chen, X. Pan, Nat. Commun. 4 (2013) 2791.

[6] A. V. Pinheiro, D. R. Han, W. M. Shih, H. Yan, Nat. Nanotechnol. 6 (2011), 763772 .

[7] W.-F. Xue, A. L. Hellewell, W. S. Gosal, S. W. Homans, E. W. Hewitt, S. E. Radford, J. Biol. Chem. 284 (2009), 34272-34282.

[8] A. H. Zewail, J. M. Thomas, 4D Electron Microscopy: Imaging in Space and Time (World Scientific Publishing, 2010).

[9] A. H. Zewail, Science 328 (2010) 187-193. 
[10] R. J. D. Miller, Science 343 (2014) 1108-1116.

[11] A. H. Zewail, 4 D Visualization of matter (Imperial College Press, 2014).

[12] V. A. Lobastov, R. Srinivasan, A. H. Zewail, Proc. Natl. Acad. Sci. U.S.A. 102 (2005), 7069-7073.

[13] B. Barwick, H. S. Park, O.-H. Kwon, J. S. Baskin, A. H. Zewail, Science 322 (2008) 1227-1231.

[14] H. S. Park, J. S. Baskin, O.-H. Kwon, A. H. Zewail, Nano Lett. 7 (2007) $2545-2551$.

[15] S. Schäfer, W. Liang, A. H. Zewail, New J. Phys. 13 (2011) 063030.

[16] R. P. Chatelain, V. R. Morrison, B. L. M. Klarenaar, B. J. Siwick, Phys. Rev. Lett. 113 (2014) 235502.

[17] R. M. van der Veen, O.-H. Kwon, A. Tissot, A. Hauser, A. H. Zewail, Nat. Chem. 5 (2013) 395-402.

[18] G. M. Vanacore, R. M. van der Veen, A. H. Zewail, ACS Nano 9 (2015) 1721-1729.

[19] S. T. Park, D. J. Flannigan, A. H. Zewail, J. Am. Chem. Soc. 134 (2012) 9146-9149.

[20] M. Gao, C. Lu, H. Jean-Ruel, L. C. Liu, A. Marx, K. Onda, S. Koshihara, Y. Nakano, X. Shao, T. Hiramatsu, G. Saito, H. Yamochi, R. R. Cooney, G. Moriena, G. Sciain, R. J. D. Miller, Nature 496 (2013) 343-346. 
[21] A. W. P. Fitzpatrick, G. M. Vanacore, A. H. Zewail, Proc. Natl. Acad. Sci. U.S.A. 112 (2015) 3380-3385.

[22] S. Schäfer, W. Liang, A. H. Zewail, J. Chem. Phys. 135 (2011) 214201.

[23] W. Liang, S. Schäfer, A. H. Zewail, Chem. Phys. Lett. 542 (2012) 1-7.

[24] D.-S. Yang, C. Lao, A. H. Zewail, Science 321 (2008) 1660-1664.

[25] R. K. Raman, R. A. Murdick, R. J. Worhatch, Y. Murooka, S. D. Mahanti, T.-R. T. Han, C.-Y. Ruan, Phys. Rev. Lett. 104 (2010) 123401.

[26] G. M. Vanacore, J. Hu, W. Liang, S. Bietti, S. Sanguinetti, A. H. Zewail, Nano Lett. 14 (2014) 6148-6154.

[27] D.-S. Yang, N. Gedik, A. H. Zewail, J. Phys. Chem. C 111 (2007) 48894919.

[28] A. Yurtsever, A. H. Zewail, Science 326 (2009) 708-712.

[29] A. Yurtsever, A. H. Zewail, Proc. Natl. Acad. Sci. U.S.A. 108 (2011) 3152-3156.

[30] W. Liang, G. M. Vanacore, A. H. Zewail, Proc. Natl. Acad. Sci. U.S.A. 111 (2014) 5491-5496.

[31] A. Yurtsever, R. M. van der Veen, A. H. Zewail, Science 335 (2012) $59-64$.

[32] H. Liu, O.-H. Kwon, J. Tang, A. H. Zewail, Nano Lett. 14 (2014) 946954. 
[33] R. F. Egerton, Electron Energy Loss Spectroscopy in the Electron Microscope (Springer, New York, 2011).

[34] F. Carbone, O.-H. Kwon, A. H. Zewail, Science 325 (2009) 181-184.

[35] R. M. van der Veen, T. J. Penfold, A. H. Zewail, Structural Dynamics 2 (2015) 024302.

[36] B. Barwick, D. J. Flannigan, A. H. Zewail, Nature 462 (2009) 902-906.

[37] D.-S. Yang, O. F. Mohammed, A. H. Zewail, Proc. Natl. Acad. Sci. U.S.A. 107 (2010) 14993-14998.

[38] O. F. Mohammed, D.-S. Yang, S. K. Pal, A. H. Zewail, J. Am. Chem. Soc. 133 (2011) 7708-7711.

[39] A. Gahlmann, S. T. Park, A. H. Zewail, Phys. Chem. Chem. Phys. 10 (2008) 2894-2909.

[40] A. Yurtsever, S. Schäfer, A. H. Zewail, Nano Lett. 12 (2012) 3772-3777.

[41] F. Bonaccorso, L. Colombo, G. Yu, M. Stoller, V. Tozzini, A. C. Ferrari, R. S. Ruoff, V. Pellegrini, Science 347 (2015) 1246501.

[42] L. Novotny, N. van Hulst, Nat. Photon. 5 (2011) 83-90.

[43] M. Schnell, A. Garcia-Etxarri, J. Alkorta, J. Aizpurua, R. Hillenbrand, Nano Lett. 10 (2010) 3524-3528.

[44] A. Yurtsever, A. H. Zewail, Nano Lett. 12 (2012) 3334-3338.

[45] B. Barwick, A. H. Zewail, ACS Photonics 2 (2015) 1391-1402. 
[46] S. T. Park, M. Lin, A. H. Zewail, New J. Phys. 12 (2010) 123028.

[47] M. T. Hassan, H. Liu, J. S. Baskin, A. H. Zewail, Proc. Natl. Acad. Sci. U.S.A. 112 (2015) 12944-12949.

[48] J. S. Baskin, A. H. Zewail, C. R. Phys. 15 (2014) 176-189.

[49] A. Yurtsever, J. S. Baskin, A. H. Zewail, Nano Lett. 12 (2012) 5027-5032.

[50] L. Piazza, T. T. A. Lumen, W. Quiñonez, Y. Murooka, B. W. Reed, B. Barwick, F. Carbone, Nat. Commun. 6 (2015) 6407.

[51] M. Urbakh, E. Meyer, Nat. Mater. 9 (2010) 8-10.

[52] D. J. Flannigan, S. T. Park, A. H. Zewail, Nano Lett. 10 (2010) 47674773.

[53] H. Yoshizawa, Y. L. Chen, J. Israelachvili, J. Phys. Chem. 97 (1993) 41284140.

[54] U. J. Lorenz, A. H. Zewail, Science 344 (2014) 1496-1500.

[55] B. Fultz, Phase Transitions in Materials (Cambridge University Press, Cambridge, 2014).

[56] P. Baum, D.-S. Yang, A. H. Zewail, Science 318 (2007) 788-792.

[57] V. R. Morrison, R. P. Chatelain, K. L. Tiwari, A. Hendaoui, A. Bruhács, M. Chaker, B. J. Siwick, Science 346 (2014) 445-448.

[58] J. Hu, G. M. Vanacore, Z. Yang, X. Miao, A. H. Zewail, ACS Nano 9 (2015) 6728-6737. 
[59] B.-K. Yoo, O.-H. Kwon, H. Liu, J. Tang, A. H. Zewail, Nat. Commun. $6(2015) 8639$.

[60] V. Niel, J. Martinez-Agudo, M. Munoz, A. Gaspar, J. Real, Inorg. Chem. 40, (2001) 38383839.

[61] J. Shah, Ultrafast Spectroscopy of Semiconductors and Semiconductor Nanostructures (Springer-Verlag, New York, 1999).

[62] E. Najafi, T. D. Scarborough, J. Tang, A. H. Zewail, Science 347 (2015) 164-167.

[63] B. Sapoval, C. Hermann, Physics of Semiconductors (Springer-Verlag, New York, 1995).

[64] J. Cho, T. Y. Hwang, A. H. Zewail, Proc. Natl. Acad. Sci. U.S.A. 111 (2014), 2094-2099.

[65] J. Cho et al., Nat. Phys. (2015).

[66] F. Carbone, Structural Dynamics 2 (2015) 020601.

[67] N. Bonini, J. Garg, N. Marzari, Nano Lett. 12 (2012) 2673-2678.

[68] D. Olguin, M. Cardona, A. Cantanero, Solid State Commun. 122 (2002) 575-589.

[69] B. Huang, H. Babcock, X. Zhuang, Cell 143 (2010), 1047-1058.

[70] E. Behrmann, J. Loerke, T. V. Budkevich, K. Yamamoto, A. Schmidt, P. A. Penczek, M. R. Vos, J. Bürger, T. Mielke, P. Scheerer, C. M. T. Spahn, Cell 161 (2015), 845-857. 
[71] D. J. Flannigan, B. Barwick, A. H. Zewail, Proc. Natl. Acad. Sci. U.S.A. 107 (2010) 9933-9937. (B)

[72] M. Adrian, J. Dubochet, J. Lepault, A. W. McDowall, Nature 308 (1984), 3236.

[73] K. A. Taylor, R. M. Glaeser, Science 186 (1974) 10361037

[74] V. Lǔcić, A. Rigort, W. Baumeister, J. Cell Biology 202 (2013), 407-419

[75] A. Brown, A. Amunts, X.-c. Bai, Y. Sugimoto, P. C. Edwards, G. Murshudov, S. H. W. Scheres, V. Ramakrishnan, Science 346 (2014), 718722 .

[76] A. Bartesaghi, A. Merk, S. Banerjee, D. Matthies, X. Wu, J. L. S. Milne, S. Subramaniam, Science 348 (2015), 1147-1151.

[77] A. W. P. Fitzpatrick, U. J. Lorenz, G. M. Vanacore, A. H. Zewail, J. Am. Chem. Soc. 135 (2013), 19123-19126.

[78] A. W. P. Fitzpatrick, G. T. Debelouchina, M. J. Bayro, D. K. Clare, M. A. Caporini, V. S. Bajaj, C. P. Jaroniec, L. Wang, V. Ladizhansky, S. A. Mller, C. E. MacPhee, C. A. Waudby, H. R. Mott, A. De Simone, T. P. J. Knowles, H. R. Saibil, M. Vendruscolo, E. V. Orlova, R. G. Griffin, C. M. Dobson, Proc. Natl. Acad. Sci. U.S.A. 110 (2013), 5468-5473.

[79] F. Chiti, C. M. Dobson, Annu. Rev. Biochem. 75 (2006), 333-366.

[80] C. Sachse, N. Grigorieff, M. Fändrich, Angew. Chem. Int. Ed. Engl. 49 (2010), 1321-1323. 
[81] S. L. Guo, B. B. Akhremitchev, Biomacromolecules 7 (2006), 16301636.

[82] T. P. J. Knowles, A. W. Fitzpatrick, S. Meehan, H. R. Mott, M. Vendruscolo, C. M. Dobson, M. E. Welland, Science 318 (2007), 19001903.

[83] J. F. Smith, T. P. J. Knowles, C. M. Dobson, C. E. Macphee, M. E. Welland, Proc. Natl. Acad. Sci. USA 103 (2006), 1580615811.

[84] A. W. P. Fitzpatrick, S. T. Park, A. H. Zewail, Proc. Natl. Acad. Sci. U.S.A. 110 (2013), 10976-10981.

[85] R. P. Friedrich, K. Tepper, R. Rönicke, M. Soom, M. Westermann, K. Reymann, C. Kaether, M. Fändrich, Proc. Natl. Acad. Sci. USA 107 (2010), 19421947.

[86] F. A. Aldaye, A. L. Palmer, H. F. Sleiman, Science 321 (2008), 17951799.

[87] U. J. Lorenz, A. H. Zewail, Proc. Natl. Acad. Sci. U.S.A. 110 (2013), $2822-2827$.

[88] D. M. Ebenstein, L. A. Pruitt, Nano Today 1 (2006), 2633.

[89] M. F. Ashby, D. R. H. Jones (2012) Engineering Materials 1: An Introduction to Properties, Applications and Design (Elsevier, Oxford), Vol 1.

[90] M. Bathe, C. Heussinger, M. M. A. E. Claessens, A. R. Bausch, E. Frey E, Biophys. J. 94 (2008), 29552964.

[91] R. Mankowsky, A. Subedi, M. Först, S. O. Mariager, M. Chollet, H. T. Lemke, J. S. Robinson, J. M. Glownia, M. P. Minitti, A. Frano, M. 
Fechner, N. A. Spaldin, T. Loew, B. Keimer, A. Georges, A. Cavalleri, Nature 516 (2014), 71-73.

[92] A. H. Zewail, Sci. Am. 303, (2010) 74. 


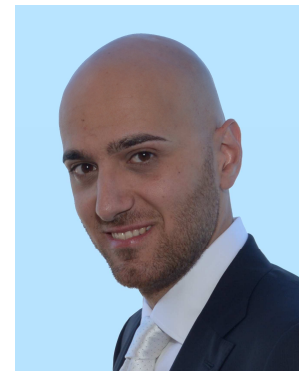

Giovanni Maria Vanacore received his BS and MS in physics engineering from the Politecnico di Milano (Italy), and his Ph.D. in physics under the cotutorship of the Politecnico di Milano (Italy), the École Polytechnique X (France), and the French Atomic Energy Commission (CEA, France). He is currently a postdoctoral fellow in the group of Prof. A H. Zewail at the California Institute of Technology (U.S.A). His current research is focused on ultrafast phenomena in condensed matter, with a special interest in electron-phonon coupling, phonon-phonon interactions, and phase transitions in low-dimensional nanoscale materials.

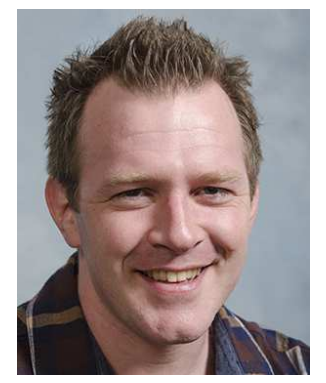

Anthony W. P. Fitzpatrick is a Marie Curie International Outgoing Fellow from the University of Cambridge (England) working on the structure of amyloid using a combination of solid-state nuclear magnetic resonance and cryo-electron microscopy. He has a biophysics background (Ph.D. with Professor Christopher M. Dobson, University of Cambridge) and did (joint) postdocs with Professors Helen Saibil in London, Robert G. Griffin at the Massachusetts Institute of Technology (U.S.A.) and Ahmed H. Zewail at the California Institute of Technology.

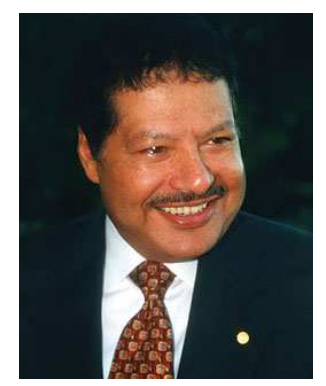

Ahmed H. Zewail is the sole recipient of the 1999 Nobel Prize in Chemistry. Currently, he is the Linus Pauling Chair Professor of Chemistry and Physics, and Director of the Center for Physical Biology at the California Institute of Technology. He has published some 600 articles and 14 books and is known for his effective public lectures and writings, not only on science but also in global affairs. For his leadership role in world affairs, he received, among others, the "Top American Leaders Award" 
from The Washington Post and Harvard University. In 2009, President Barack Obama appointed him to the Council of Advisors on Science and Technology, and in the same year he was named the first U.S. Science Envoy to the Middle East. Following the 2011 Egyptian revolution, the government established the "Zewail City of Science and Technology" as the national project for scientific renaissance, and Dr. Zewail became its first Chairman of the Board of Trustees. 